\title{
An interplay between the p38 MAPK pathway and AUBPs regulate c-fos mRNA stability during mitogenic stimulation
}

Maria Sol Degese ${ }^{*} \dagger$, Tamara Tanos ${ }^{*} \dagger$, Julian Naipauer ${ }^{*} \dagger$, Timothy Gingerich $\ddagger$, Diego Chiappe§, Pablo Echeverria\|, Jonathan LaMarreł J. Silvio GutkindTा \& Omar A. Coso* ${ }^{1}$.

* LFBM-DFBMC, Departamento de Fisiología y Biología Molecular, Facultad de Ciencias Exactas y Naturales, Universidad de Buenos Aires (FCEN-UBA), Buenos Aires C1428EHA, Argentina.

† Instituto de Fisiología, Biología Molecular y Neurociencias (IFIBYNE-FCEN-UBA-CONICET), Buenos Aires, Argentina.

‡ DBMS, Ontario Veterinary College, University of Guelph, Guelph, ON, Canada, N1G 2W1.

$\S$ Proteomics Core Facility, École Polytechnique Fédérale de Lausanne, CH-1015 Lausanne, Switzerland.

|| Département de Biologie Cellulaire, Université de Genève, Sciences 3, CH - 1211 Genève 4, Switzerland.

IT Oral and Pharyngeal Cancer Branch, National Institute of Dental and Craniofacial Research, $\mathrm{NIH}$, Bethesda, MD 20892-4330, USA.

1 Corresponding Author

Running title: MAPK regulation of fos mRNA decay. 


\begin{abstract}
MAPK pathways constitute key regulatory elements linking extracellular stimuli to nuclear gene expression. Immediate-early responsive genes of the AP-1 family, as fos, achieve peak expression levels shortly after cells are stimulated with growth factors, and sharply decrease thereafter. Several AU-rich binding proteins (AUBPs), including HuR and KSRP, bind to a fos ARE element present in the 3'UTR region of fos mRNA regulating its stability by a still poorly defined mechanism. We show here that while HuR binds and stabilizes transcribed reporter mRNAs bearing the fos 3'UTR, KSRP counteracts this effect. Furthermore, we found that fos mRNA stability and HuR phosphorylation status are dependent on the activity of p38 MAPK in both epithelial cells and fibroblasts upon proliferative stimulation. Analyzing PPI (protein-protein interaction) networks, we performed a thorough query of interacting proteins for p38 MAPKs, HuR and other AUBPs upon growth factor stimulation. This revealed novel HuR interactors, including inhibitors to PP2A activity. Over-expression of two of these interactors, pp32 and APRIL and pharmacological inhibition of PP2A stabilized a fos reporter mRNA. Our results indicate that p38 MAPK regulates fos mRNA decay by affecting the state of phosphorylation of HuR while controlling, yet to be fully elucidated, protein phosphatase regulatory networks.
\end{abstract}




\section{Introduction}

Immediate-early responsive genes (IEGs) are rapidly and transiently induced in response to a variety of stimuli in living cells. Members of the AP-1 family of transcription factors (composed mainly of the c-jun subfamily and c-fos related genes) are among its most conspicuous representatives [1]. Particularly for the c-fos gene (referred as fos herein), its mRNA level peaks shortly after 30 minutes of stimulation, and decreases rapidly afterwards $[2,3]$.

MAP (mitogen activated protein) kinases are key components of important signaling pathways that transduce extracellular signals and ultimately regulate gene expression. Regulation of fos mRNA levels and protein activity are regulated by mechanisms involving MAP kinases signaling pathways $[1,3,4]$. While the fos promoter exhibits a complex array of regulatory elements targeted by different transcription factors, the c-Fos (Fos) protein contains a number of serine and threonine residues that represent targets for phosphorylation and concomitant transcriptional regulation [1,4-6].

Besides regulation of promoter and protein activity, post-transcriptional decay of fos mRNA provides one more regulatory instance for the fos gene family. AU-rich (adenine and uracil) elements (ARE) are present in the mRNA of fos (and many other IEGs), which are essential for the regulation of mRNA decay processes $[7,8]$. ARE sequences are believed to be the main docking regions for most proteins involved in regulating mRNA stability [7]. There are several types of ARE regions, mostly characterized by the presence and repetition of the consensus sequence AUUUA; specifically fos has a well characterized class I ARE in its 3'UTR (3' untranslated region) $[9,10]$.

Trans-acting factors associated to the AREs are known as AU-binding proteins (AUBPs). Some of the better studied AUBPs are ELAVL1 or HuR (and Hu family members HuB, HuC, HuD) [11], HNRNPD or AUF1 or [12], KHSRP (KSRP) [13], ZFP36 (TTP) [14] and ZFP36L1 (BRF1) [15] among others. Some of them are involved in mRNA stabilization (HuR), while others stimulate its degradation (TTP, KSRP, BRF1, AUF1). KSRP has been shown to bind to AREs of unstable mRNAs, associate to TTP and together recruit the exosome complex, leading to mRNA degradation [16]. KSRP and TTP were reported to be phosphorylated by p38 MAPK family members (p38 $\beta$ and $p 38 \alpha$ respectively) and by the MAPK activated protein 2 (MK2) [1719]. Phosphorylated forms are unable to bind to their target sequence in mRNAs, provoking a delay of the degradation process, reversed by phosphatase reactivation. On the other hand, HuR binds to AREs and, when overexpressed, stabilizes mRNAs preventing their degradation [20]. HuR is also reported to be targeted by different kinases, such as Chk2, CDK1, PKC $\delta$ and p38a [21-24]. Phosphorylation of different residues within the sequence of HuR (S88, T118, S202, S221 and S318) is involved in increased nucleo-cytoplasmic shuttling and RNA binding [21-24].

AUBPs reported to associate to the fos ARE in different cellular contexts include HuR, HuB, HuD, AUF1 and KSRP [8]. Although several reports studied signaling pathways regulating the activity of these proteins and the fos mRNA decay process, details regarding the regulatory mechanisms in which they participate remain elusive.

In the present article, we focus on fos mRNA decay regulation by MAPKs in cultured cells under proliferative stimulation. We found that activation of the p38 MAPK pathway promotes the decay of the fos mRNA through the regulation of regulatory sequences that require an intact ARE element in its 3'UTR. We also observed that HuR binds to the fos ARE, stabilizing the fos mRNA. On the other hand, KSRP counteracts this effect, promoting fos mRNA degradation. Although we observed that p38 MAPK affects HuR mRNA binding and stabilizing properties, we did not detect a direct phosphorylation of HuR by p38 MAPK. we found that p38 MAPK activation affects HuR serine phosphorylation state in a negative fashion, 
diminishing its ability to bind to the fos ARE and promoting fos mRNA decay, possibly due to the activation of phosphatases acting on HuR. 


\section{Materials and Methods}

\section{Culture and treatments of Cell Lines}

HEK293 and HeLa TetOff (Clontech, Madison, WI) cells were maintained in Dulbecco's modified Eagle's medium high glucose (DMEM) (Invitrogen, Grand Island, NY), with L-glutamine and sodium pyruvate supplemented with $10 \%$ fetal bovine serum and penicillin/streptomycin/amphotericin B (Invitrogen, Grand Island, NY). NIH3T3 mouse fibroblasts and NIH3T3 expressing the human M1 muscarinic acetylcholine receptor ( $\mathrm{m} 1.2 \mathrm{cells}$, described in [3]) were grown in Dulbecco's modified Eagle's medium high glucose, with L-glutamine, containing $10 \%$ calf serum or bovine growth serum supplemented calf (Thermo-Scientific, Waltham, MA) and the above antimicrobial mixture. Before the stimuli, cells were starved for 2, 12 or $24 \mathrm{hrs}$, as indicated, and then treated with human recombinant PDGF-BB $100 \mathrm{ng} / \mathrm{mL}$ final concentration (Beta Laboratories, Argentina) for the NIH3T3 cell line, or EGF $10 \mathrm{ng} / \mathrm{mL}$ final concentration, in the case of HEK293 cells. The MAPK inhibitors (PD98059 at final concentration of $20 \mu \mathrm{M}, \mathrm{UO} 126$ at $10 \mu \mathrm{M}$ and SB203580 at $10 \mu \mathrm{M}$ ) and MAPKAPK2 inhibitor or MK2ai (also known as CMPD1, at $50 \mu \mathrm{M}$ ) (Calbiochem, San Diego, CA) were pre-incubated for $1 \mathrm{hr}$ before stimulation. PP2A and PP1 inhibitor Endothall (at final concentration of $50 \mu \mathrm{M}$ ) was added to the medium from 1 to 2 hs before treatment. For reporter mRNA decay assays, cells were incubated with tetracycline (Sigma-Aldrich, Saint Louis, MO) at a final concentration of 1 $\mu \mathrm{g} / \mathrm{ul}$ for a maximum of $5 \mathrm{hrs}$.

\section{Transient Transfections}

All cell lines were plated in complete media and allowed to grow overnight to $70-80 \%$ confluence. The cells were transfected using linear polyethylenimine/PEI (Polysciences, Warrington, PA) with a protocol adjusted for each cell line. Transfection complexes were achieved using the appropriate volume of PEI $(1 \mu \mathrm{g} / \mu \mathrm{l} \mathrm{pH} 7.2)$, in a 3:1 ratio (PEI:DNA) for HEK293 and HeLa TetOff cells or in a 7:1 ratio for NIH3T3 cells.

\section{DNA Constructs}

The PCR fragment corresponding to the entire 3'UTR of the murine fos mRNA (GenBank® accession number NM_010234, from 1283 to 2091 bp) was amplified from cDNA of PDGF stimulated NIH3T3 cells with appropriate primers and subcloned into the pTRE2hygLuc plasmid (Clontech, Madison, WI) using the Nhel and Clal restriction sites (Luc-fos). The 3'UTR $\triangle$ ARE of fos was obtained by deletion of $69 \mathrm{bp}$ of the described AU-rich region or ARE (Luc-fos $\triangle A R E$ ). This deletion was achieved by amplification of both regions up- and downstream of the ARE region of the fos 3'UTR from Luc-fos, and ligating them with an Agel restriction site, replacing the $69 \mathrm{bp}$ with $6 \mathrm{bp}$ of this site. pTRE2hygLuc with the entire 3'UTR of $\beta$-Globin mRNA was used as control (Luc- $\beta$ Globin). The ARE region, plus $100 \mathrm{bp}$ downstream, was subcloned into the pBluescriptSK(+) using the restriction sites Nhel and Hindlll. A fragment of equivalent size from $\beta$-globin was subcloned in the same vector and used as a control. Labeled in vitro transcripts obtained from these vectors were used in the experiments of RNA Gel mobility shift assays. AUBP expression vectors were engineered as follows: cDNA of murine HuR, TTP and BRF1 were subcloned into the following plasmids pCEFL-HA (mHuR) and pGEX4T3 (GSTHuR, GST-TTP and GST-BRF1). The cDNA encoding human HuR in its wild type version and mutant in 3 phosphorylation sites (T118A, S202A,S221A) were a gift from Angel Nebreda and were subcloned in the pCEFL-HA (hHuR WT and hHuR 3xM). For expression of Strep tagged human HuR, the pcDNA3Zeo(+)Strep2HuR was used. Expression vectors for pCEFL HAtagged JNK1, ERK2, ERK5, p38a, p38ß, p38y, and p38ס MAPK, and pGEX4T3-ATF2 have been described previously [1]. pET15b-KSRP (His-KSRP) and pcDNA4TOEGFP-KSRP [25] were gifts from Hartmut Kleinert. pcDNA3-FLAG-pp32 and pcDNA3-FLAG-APRIL were a gift 
from Joan Steitz [26].

\section{Reporter mRNA decay assays}

The quantitative PCRs to measure luciferase mRNA were performed as in [27]. The primer set used was the following: Forward 5'-ccgccgttgttgttttg-3'; Reverse 5'-acacaactcctccgcgc-3'. The amount of luciferase mRNA was normalized using primers for the hygromycin gene, present in the reporter plasmid: Forward 5'-ggaatccccgaacatcg-3'; Reverse 5'-gcagacgcgctacttcg-3'.

\section{Northern Blots and Real time qPCR assays}

Total cellular RNA was isolated using TriZol (Invitrogen, Grand Island, NY, Grand Island, NY) or TriReagent (Genbiotech, Argentina) using the manufacturer's protocols. mRNAs were reversetranscribed using oligo-dT primers (Biodynamics, Argentina). Real-time quantitative PCR was performed using a Stratagene MX 3000P QPCR with SYBR green detection (Agilent, Santa Clara, CA). For real time qPCR, $2 \mu \mathrm{g}$ of total RNA was subjected to reverse transcription. A dilution of all samples was then used to measure the expression of the endogenous fos, using a GAPDH product as normalizer. For Northern blots, $30 \mu \mathrm{g}$ of RNA extracted were ran in a $1 \%$ agarose gel in TAE 1x, transferred to a positively charged nylon membrane (HyBond, GE Healthcare, Wauwatosa, WI) and incubated with a fos specific probe $\left[{ }^{32} \mathrm{P}\right]$ labeled by PCR.

\section{RNA Electrophoretic Mobility Shift Assays}

RNA probes were synthesized with a T7 RNA transcription Kit (Promega, Madison, WI) from the pBSfosARE as well as the $\beta$-globin control. For radioactive probes [ $\left.\alpha-{ }^{32} \mathrm{P}\right] \mathrm{rUTP}$ (NEN Perkin Elmer, Waltham, MA), was added. Total extracts were obtained from NIH3T3 cells plated in 6$\mathrm{cm}$ plates and grown to $90 \%$ confluence, starved overnight, and then treated with PDGF 10 $\mathrm{ng} / \mathrm{ml}$ and pretreated (or not) with SB 203580 and other inhibitors as indicated. Protocol for protein extraction and probe incubation have been previously described [28].

\section{Solid phase kinase assays}

NIH3T3 cell were incubated in $6 \mathrm{~cm}$ plates to $80 \%$ conlfuence, starved for $12 \mathrm{hrs}$ and stimulated with PDGF for different times as previously described. The plates were washed with cold phosphate-buffered saline, and lysed at $4^{\circ} \mathrm{C}$ in a buffer containing $25 \mathrm{mM} \mathrm{HEPES}, \mathrm{pH} 7.5,0.3$ $\mathrm{M} \mathrm{NaCl}, 1.5 \mathrm{mM} \mathrm{MgCl}_{2}, 0.2 \mathrm{mM}$ EDTA, $0.5 \mathrm{mM}$ DTT, $1 \%$ Triton X-100, 0.1\% SDS, $10 \mathrm{mM} \beta-$ glycerophosphate, $1 \mathrm{mM}$ sodium vanadate, and $1 \mathrm{mM}$ PMSF. A mix of Glutathione-sepharose beads (GE Healthcare, Wauwatosa, WI) with bound GST-HuR was added corresponding to 4 $\mu \mathrm{g}$ of protein to each tube, and incubated at $4^{\circ} \mathrm{C}$ overnight. The following day the beads were collected and washed and developed as described for the kinase assay protocol in vitro.

\section{Western Blot Analysis}

The protocol is described elsewhere [27]. The primary antibodies used were the following: antiKSRP (\#5398, Cell Signaling Technologies, Danvers, MA), anti-HuR (sc-5261, Santa Cruz Biotechnologies, Santa Cruz, CA), anti-c-Fos (sc-7202, Santa Cruz Biotechnologies, Santa Cruz, CA), anti-Actin (sc-1616, Santa Cruz Biotechnologies, Santa Cruz, CA), anti- $\beta$ Tubulin (sc9935, Santa Cruz Biotechnologies, Santa Cruz, CA), anti- $\alpha$ Tubulin (\#3873, Cell Signaling Technologies, Danvers, MA), anti-HA (sc-805, Santa Cruz Biotechnologies, Santa Cruz, CA), anti-GFP (sc-8334, Santa Cruz Biotechnologies, Santa Cruz, CA) anti-FLAG (F7425, SigmaAldrich, Saint Louis, MO-Aldrich) and anti-pSer (\#05-1000, Millipore, Billerica, MA), anti-ERK2 (sc-154-G, Santa Cruz Biotechnologies, Santa Cruz, CA), anti-ERK5 (\#3372 Cell Signaling Technologies, Danvers, MA), anti-p38 $\alpha$ (\#9212 Cell Signaling Technologies, Danvers, MA), anti-p38 $\beta$ (sc-6176 Santa Cruz Biotechnologies, Santa Cruz, CA), anti-p38y (sc-6022 Santa Cruz Biotechnologies, Santa Cruz, CA), anti-p38ס (sc-7585 Santa Cruz Biotechnologies, Santa 
Cruz, CA).

\section{Biotin-RNA pull-down assays}

5 ' end biotin labeled RNA oligos (Bi-RNA) were used to isolate proteins that associate with the ARE of fos. The following probes were used: 1) ARE-WT oligo, $40 \mathrm{bp}$ long that includes the 3 AUUUA consensus sequences (Dharmacon Thermo-Scientific, Waltham, MA) ; 2) MUT3x, also $40 \mathrm{bp}$ long and has the 3 uracils of AUUUA mutated to guanidine (Dharmacon ThermoScientific, Waltham, MA); 3) negative control, non ARE RNA sequence from the EDI gene 47 bp long (Dharmacon Thermo-Scientific, Waltham, MA). Lysates from HEK293 and NiH3T3 cells were quantified, precleared with Strepavidin agarose beads (Thermo-Scientific, Waltham, MA) and $100 \mu \mathrm{g}$ of protein was incubated in the presence of $0.5 \mathrm{nmol}$ of each probe for 10 minutes at RT, and 10 minutes at $4^{\circ} \mathrm{C}$. After several washes, SDS-sample buffer was added and a SDSPAGE was performed. The gels were either stained or transferred and subjected to Western Blot analysis.

\section{Identification of proteins by LC-MS}

To determine protein identity, after the isolation of Bi-RNA and bound protein complexes, the proteins were separated by SDS-PAGE. Each gel was fixed and stained using either Pierce Silver Stain kit (Pierce, Rockford, IL) or colloidal commassie. After the staining protocol, the bands were sliced. Stained gel pieces were first washed and then reduced-alkylated (DTElodoacetamide). Proteolytic digestion was performed overnight at $37^{\circ} \mathrm{C}$ using modified Trypsin (Promega, Madison, WI) in $50 \mathrm{mM}$ ammonium bicarbonate $(\mathrm{pH} 8.3)$. Peptides were then extracted from gels and concentrated by Speed Vac prior to LC-MS measurements. Dried samples were resuspended in LC-MS loading solvent (2\% Acetonitrile, $0.1 \%$ formic acid) and separated by HPLC (nanoAcquity, Waters, Milford, MA). Samples were first captured and washed at initial chromatographic conditions on a home-made capillary Pre-column (Magic C18; $3 \mu \mathrm{m}-200 \AA ; 2 \mathrm{~cm} \times 100 \mu \mathrm{m})$ prior to analytical separation. An $80 \mathrm{~min}$ biphasic gradient was run starting from $100 \%$ A solvent (2\% Acetonitrile, $0.1 \%$ formic acid) to $90 \%$ B solvent $(100 \%$ acetonitrile, $0.1 \%$ formic acid) on a home-made capillary column (Magic C18; $3 \mu \mathrm{m}-100 \AA ; 15 \mathrm{~cm}$ $x 75 \mu \mathrm{m}$ ID at $250 \mathrm{nl} / \mathrm{min}$ ). Online mass spectrometric detection was performed on an LTQOrbitrap XL (Thermo-Scientific, Waltham, MA) using Data Dependent Acquisition mode with dynamic exclusion. For each MS scan, the ten most intense detected ions were fragmented and then excluded for the following 30 seconds. Experimentally generated data was submitted to protein database search through Proteome Discoverer 1.1 (Thermo-Scientific, Waltham, MA) and Mascot 2.3 (Matrix Science) search engine against the Human restricted Uniprot Protein database (Uniprot release 2011_07- May 31, 2011 including its reversed format). Scaffold 3 Viewer (Proteome Software) was used to finally compile data results.

\section{Bacterial Expression of GST Fusion Proteins}

The BL21 pLysS strain of E. coli was transformed with the vector pGEX-4T3 encoding the fusion protein GST-mHuR WT and with pET15b-KSRP (His tagged KSRP). The protocol used to purified recombinant proteins was described in [1].

\section{SIRNA}

$\mathrm{NIH} 3 \mathrm{~T} 3$ were transfected with HiPerfect (Qiagen, Germany) with a siRNA oligo targeting HuR sequence (Qiagen, Germany) and AllStars oligo as negative control (Qiagen, Germany). 48 hrs post transfection cells were starved and stimulated with PDGF and lysed to analyze fos and HuR protein expression as indicated.

\section{Luciferase Reporter Assays}


Cells were seeded on 6-well or 12-well dishes and transfected with different expression plasmids together with $0.1 \mathrm{ug}$ of luciferase fos promoter reporter vector. The total amount of transfected DNA was normalized with $\beta$-galactosidase assay. Cells were lysed in passive lysis buffer (Promega, Madison, WI) 24 hrs posttransfection. Cell lysates were incubated with luciferin reagent of the luciferase Reporter System (Promega, Madison, WI).

\section{Network-based data organization and discovery.}

Integration of experimental data in protein-protein interaction (PPI) networks to build explorable maps using Cytoscape (http://www.cytoscape.org) has been previously described [29,30]. Briefly, using the human-centered PPI database built by Echeverria and colleagues [31] it was possible to extract a network containing the PPls between the query proteins and their interactors. Query proteins used in the process were: p38 alpha (MAPK14), p38 beta (MAPK11), p38 gamma (MAPK12), p38 delta (MAPK13), MK2 (MAPKAPK2), TTP (ZFP36), HuR (ELAVL1), BRF1 (ZFP36L1), KSRP (KHSRP) and AUF1 (HNRNPD). The set of PPI was further enriched by manually curated literature mining. One "node" in this network corresponds to a protein and the connection between them is called "edge" and it refers to the PPI between these two nodes. Information for the different members of the network was also loaded from different databases (Uniprot, Gene Ontology) to include in the graph as metadata. These efforts were then complemented by literature mining to improve our understanding of the potential biological relevance of these connected modules.

\section{TUNEL assay.}

The percentage of apoptotic cells was measured using the ApopTag Fluorescein In Situ Kit (Millipore, Billerica, MA). Cells were plated into glass slides and, after treated as indicated, fixed in paraformaldehyde $1 \%$ for 10 minutes. DNA fragmentation was detected according to manufacturer's instructions. The Kit uses DNA terminal deoxynucleotidyl transferease (TdT) to catalyze the addition of digoxynenin-dNTP to $3^{\prime}$ ends of fragmented DNA. This tagged tail is finally detected with a specific fluorescein tagged antibody. The slides were counterstained with DAPI, several pictures were taken for each treated sample using a Zeiss Axioimager.Z1 with Apotome attachment. At least 1000 cells were quantified in each treatment. Positive cells were quantified using the ImageJ software and percentage of apoptotic cells was determined.

\section{Statistical Analysis}

All experiments were repeated at least 3 times. The statistical significance was measured with one-way ANOVA test and multiple mean comparisons in GraphPad Prism 5 software. The symbols are informed as follows: ns: not significant difference; *: significant difference of the means when $\alpha=0.05$ is considered; ${ }^{* *}$ : $\alpha=0.01 ;{ }^{* *}: \alpha=0.001$. 


\section{$\underline{\text { Results: }}$}

Regulation of fos mRNA expression by MAPKs.

As an immediate-early responsive gene, fos shows a pattern of rapid and transient expression of its mRNA upon the addition of proliferative stimuli to cultured cells. To begin exploring the mechanism of fos expression regulation, we incubated NIH3T3 cells with Carbachol or PDGF. While the latter acts on endogenously expressed tyrosine kinase growth factor receptors, the former acts on M1 G protein coupled receptors, which transduce potent mitogenic signals when ectopically expressed in NIH3T3 cells [3], thus enabling us to study the shared key elements activated by distinct growth promoting factors. Northern blot and real time qPCR analysis showed that these proliferative stimuli enhance fos mRNA levels rapidly in NIH3T3 cells, which peaked around 30 minutes after treatment, and quickly went down within 2 hs post-stimulation (Figure $1 \mathrm{~A}$ and $1 \mathrm{~B}$ ). In an attempt to identify signaling pathways involved, we first tested for kinases involved in the regulation of fos promoter activity. We performed luciferase reporter assays cotransfecting $\mathrm{NIH} 3 \mathrm{~T} 3$ cells with a reporter containing the luciferase gene downstream the fos promoter along with vectors that express MAPKs and its upstream activating MAPKKs (Figure 1C). All kinases were expressed at comparable levels (Supplementary Figures $1 \mathrm{~A}$ and $1 \mathrm{~B}$ ) and we found that only activation of the ERK2 signaling pathway influences fos promoter activity. Similar results were obtained in a slightly different environment, when cells were cotransfected with the reporter and MAPK expression vectors, and then treated with Carbachol or PDGF. As shown in Figure 1D, neither ERK5, JNK nor any of the p38 MAPKs affected fos promoter activity to the extent induced by ERK2.

To study this using a complementary pharmacological approach, NIH3T3 cells were pretreated with MAPK inhibitors PD98059 (inhibits MEK activity, an ERK2 activator) and SB203580 (inhibits p38a MAPK activity) and stimulated with Carbachol for 40 min, which stimulates all MAPKs in these cells (Supplementary Table 1). Figures $2 \mathrm{~A}$ and $2 \mathrm{~B}$ show that in Northern Blots and GPCR assays, inhibition of the ERK2 pathway led to a decreased level of fos mRNA expression. However, after inhibiting p38 MAPK signaling, we found a significant increase in the amount of fos mRNA comparing to control conditions. Stimulating cells with PDGF rendered comparable results (Figure 2B, right panel). An alternative MEK inhibitor was tested (UO126) and rendered identical results (Supplementary Figure 2A). None of these kinase inhibitors triggered apoptosis to a significant level under the conditions used (Supplementary Figure 2B).

\section{The p38 MAPK pathway regulates fos mRNA decay}

In order to study the effects of p38 signaling pathway on post-transcriptional events, we focused on elements present in the fos mRNA structure (Figure $3 \mathrm{~A}$ ) that may affect its stability or decay. Figure $3 \mathrm{~A}$ shows a schematic representation of the murine fos mature mRNA structure, highlighting a 69 base pair region (ARE) reported to be the main docking sequence for most proteins involved in regulating mRNA stability [9]. To address the regulatory mechanisms in the mRNA decay process and given the potential importance of this region, we cloned the entire fos 3' UTR downstream luciferase to generate a different reporter construct. The new reporter plasmid has a promoter that is responsive to the addition of tetracycline (TetOff), allowing the specific regulation of the reporter gene. We engineered two reporter constructs, Luc-fos containing the entire 3' UTR of fos wild type mRNA (Figure 3B) and Lucfos $\triangle A R E$ containing the fos 3'UTR with the ARE region deleted and replaced by an Agel 
restriction site. An additional control is provided by Luc- $\beta$ globin, a reporter that expresses the luciferase gene fused to the 3' UTR region of the $\beta$-globin gene.

Upon transfection of HeLa TetOff cells with the reporter vectors, tetracycline was added to the media and cells lysed following a time course. Reporter mRNA levels were then tested by qPCR. Results show that Luc-fos produces a message that is unstable (Figure 3C). After $5 \mathrm{hrs}$ of transcription inhibition we could detect only about 20 percent of the original amount of luciferase mRNA. In contrast, mRNA expressed by control plasmids (both missing ARE elements) show no significant decay even $5 \mathrm{hrs}$ after transcription was interrupted.

Cotransfection of the Luc-fos reporter construct with MEK3EE and MEK3AA (constitutively active and dominant negative forms of the dual p38 MAPKK, MEK3) revealed that the inhibition of the p38 MAPK signaling pathway prevents reporter mRNA decay (Figure 3D). Furthermore, treatment with the inhibitor SB203580 also prevents the decay of the WT reporter (Figure 3E left panel). In order to test for a direct or indirect effect of p38 MAPK in the regulation of this post-transcriptional process by p38 MAPK, we tested MK2ai, an inhibitor targeting MAPKAPK2a (MK2 $\alpha$ ), a downstream target of p38 MAPK. MK2ai also prevented the decay of the Luc-fos reporter, suggesting a possible role for this kinase in the fos mRNA decay process (Figure 3E left panel). Neither inhibitor had any detectable effect upon the decay of reporter control mRNAs (Figure 3E, middle and right panels).

Following the time course expression profiles for endogenous fos mRNA in NIH3T3 cells stimulated with PDGF, we found that treatment with these two pharmacological inhibitors rendered a curve with delayed decay. We quantified levels of mRNA present at 30 and 60 min after the addition of PDGF in cells treated with vehicle or inhibitors for p38 MAPK and MK2. Figure $3 \mathrm{~F}$ shows the ratio of the fos mRNA present at $60 \mathrm{~min}$ vs $30 \mathrm{~min}$ after stimulation for each treatment. Incubation with both kinase inhibitors resulted in a several fold increase in this ratio with respect to control conditions. These results indicate that fos mRNA decay may require active p38 MAPK signaling.

\section{AUBPs and AUBPs interacting proteins are linked to the ARE region of fos $m R N A$}

In an attempt to identify the proteins that may link the p38 MAPK pathway to the decay fos messenger RNA we performed biotin-RNA pull-down assays. A 40 bp long 5' biotinylated RNA probe with nucleotide sequence corresponding to the WT fos ARE including all three consensus AUUUA pentamer sequences was commercially synthesized. In addition a control mutant probe with each core AUUUA sequence replaced by AGGGA was prepared. Proteins obtained from cell lysates were incubated with the probes, unbound material washed and retained proteins ran on PAGE-SDS gels (Figure 4A). Proteins present in the recovered proteinRNA complexes were subsequently identified by high resolution LC-MS. This analysis rendered a list of putative ARE associated factors as shown in Supplementary Table 2. Of interest, most of these proteins exhibit RNA binding domains, and four of them are known ARE binding proteins (AUBPs): HuR, HuB, AUF1 and KSRP. Comparing the information obtained with the alternative probes, these AUBPs preferentially associated to the WT rather than the mutant probes (Figure 4A and Table 2). We validated this information by performing Western blots using material pulled down with both fos probes and an unrelated sequence. HuR and KSRP bind only to the ARE sequences and mostly to the WT probe, with limited affinity for the mutant version (Figure 4A, lower panels).

We next used systems biology approaches to provide additional predictive information that may help understand the potential relationship between p38 MAPKs, AUBPs binding to the 
fos ARE, and putative additional interacting molecules. Organizing and integrating proteinprotein interaction (PPI) data from public sources in biological maps allowed us to explore new features in signaling systems using network-based discovery methodologies [31], using a previously reported approach [29-31]. These proteins included all 4 isoforms of p38 MAPK ( $\alpha, \beta$, $Y$ and $\delta$ ), the MAPKAPK2 or MK2, and all AUBP reported in the literature to bind to fos ARE: HuR, KSRP, AUF1 and TTP [8] and a non-associating AUBP, BRF1. Figure 4B shows one possible result of this analysis. Only KSRP was reported to interact physically with one isoform of p38 MAPK ( $\beta$ ) [32]. The network prediction analysis revealed that KSRP and AUF1 relate to many mRNA degradation proteins such as exosome complex members (EXOCS2, EXOCS3, $P A R N, D C P 2, E X O C S 4$ ), regulators of the deadenylation and the translationally coupled mRNA decay process (PABPC1, PAIP1, IGF2BP2, CSDE1, SYNCRIP, YBX1) or other decay related AUBPs (TTP). It is also interesting that these two AUBPs could be part of the same type of complex as they are connected by 2 hnRNP proteins (HNRNPA1 and HNRNPH1). On the other hand, interactors of HuR seem to be related to proteins regulating phosphatase activity, as shown in the non-characterized predicted interaction with a regulatory subunit of the phosphatase PP2A (PPP2R2B) and the interactions with SET, pp32 and APRIL, three PP2A inhibitors [33]. In grey we highlight those interactors that were found experimentally in the pulldown studies of biotinylated-RNA probe. Proteins pinpointed by both the pull-down assay using a fos ARE probe as bait and the bioinformatics approach represent putative candidates as part of a regulatory mechanism linking protein kinase signaling to fos mRNA stability.

We next cotransfected a plasmid that expresses murine HA tagged HuR (mHuR) with each luciferase-3'UTR reporter. Figure 5A shows that overexpression of HuR prevents decay of the Luc-fos reporter (left panel) while no effect is observed over the decay pattern of control reporters (middle and right panel, insert shows effective expression of HuR). In addition, knock down of HuR expression has a clear detrimental effect on endogenous fos protein expression (Figure 5B).

Protein phosphorylation is a common feature to mechanisms controlling gene expression. We tested the effect of a triple HuR mutant (T118A, S202A, and S221A) missing critical residues reported to be the target of different kinases [21-24]. Using reporter plasmids cotransfected with an HA-tagged human HuR triple mutant (HuR 3xM) or the wild type control (hHuR WT) we challenged the Luc-fos reporter (Figure 5C). In contrast to wild type HuR, the triple mutant was unable to stabilize the reporter.

Phosphatase 2 A (PP2A) inhibitor proteins, pp32 and APRIL, are also HuR interactors [33] and we tested their effect upon mRNA stability in fos reporter assays. As shown in Figure $5 D$, cotransfection with plasmids expressing either pp32 or APRIL significantly stabilized wild type fos reporter mRNA. To ascertain the importance of phosphatase activity in the regulation of the decay of fos mRNA, we incubated cells transfected with wild type reporters and treated them with Endothall, a PP2A and PP1 pharmacological inhibitor. Results as shown in Figure 5E, indicate that two hours after transcription is inhibited reporter mRNA levels drop to $50 \%$. However, pre-incubation with Endothall show no decay effect compared to control conditions strengthening the idea that phosphatase inhibition has a stabilizing effect upon fos mRNA.

We also tested the influence of KSRP on the decay experiments using two different experimental settings. Figure $5 \mathrm{~F}$ shows that in cells that are transfected with the Luc-fos reporter, overexpression of KSRP counteracts the stabilization effects elicited by SB203580 (in Figure $3 \mathrm{E}$ ) and HuR (in Figure $5 \mathrm{~A}$ and $5 \mathrm{C}$ ). As seen on Figure $5 \mathrm{C}$, phosphorylation of HuR would be necessary to perform its stabilizing effect. Therefore we tested the hypothesis that p38 MAPK might be affecting the phosphorylation status of HuR. Solid-phase kinase assays were 
performed using glutathione beads loaded with bacterially expressed GST-HuR recombinant protein. Cells treated with PDGF and SB203580 or vehicle were lysed, incubated with the beads and tested for kinase activity. Figure $6 \mathrm{~A}$ show representative gels and Figure $6 \mathrm{~B}$ correspond to bar graphs obtained after quantification of bands from three independent experiments. Treatment with the p38 MAPK inhibitor resulted in a significant increase of HuR phosphorylation that is maximal $60 \mathrm{~min}$ after the addition of PDGF. Similar results were obtained by overexpression of a Strep-tagged HuR in EGF stimulated HEK293 cells pretreated with vehicle or SB203580, to extend our analysis to other cell types. Western blots revealed with an antiphospho-Serine antibody showed an increment in the levels of phospho-Serine of HuR in the absence of p38 MAPK activity (Figure 6C).

Gel electromobility shift assays (EMSAs) can help reveal protein complexes bound to nucleic acids. In order to investigate the identity and dynamics of proteins binding to the fos ARE we performed RNA EMSAs using $\left[{ }^{32} \mathrm{P}\right]$-labeled transcripts containing the fos ARE sequence. Figure 7A shows the main delayed complexes observed, named complex 1 (C1) and complex 2 (C2). They are composed of protein bound to the labeled probe as they disappear upon proteinase $\mathrm{K}$ treatment (PK, Figure 7A). Competing with unlabeled fos ARE or $\beta$-globin oligonucleotides for complex assembly show specificity for fos ARE sequences (Figure 7A). Labelled fos ARE probe challenged with recombinant bacterially expressed AUBPs show that only HuR and KSRP seem to bind directly to the ARE (Figure 7B). Supershift assay performed using lysates from cells stimulated with PDGF pre-incubated with specific antibodies for the indicated proteins support the idea of HuR binding to the fos ARE as a major component of C2 complexes (Figure 7C).

Over a time course performing EMSAs using lysates of cells treated with PDGF we observed that C2-HuR complex levels increase after addition of the stimulus, while intensity of bands corresponding to $\mathrm{C} 1$ complexes concomitantly decrease, both effects being maximal at $30 \mathrm{~min}$, then C2 levels start to decrease and C1 levels augment, restoring original values after 2 hrs (Figure 7D). A similar experiment performed with the addition of SB203580 and quantifying bands corresponding to the $\mathrm{C} 2-\mathrm{HuR}$ complex show a statistically significant increase in the levels of C2-HuR complex formation in the presence of the p38 MAPK inhibitor (Figure 7E). 


\section{Discussion:}

As a representative early responsive gene, fos is rapidly and transiently induced upon the onset of environmental conditions that imply changes in cell fate commitment. In resting cells fos expression is maintained at very low levels. Upon stimulation of cells with growth factors acting on either tyrosine kinase or G-protein coupled receptors the fos promoter is rapidly induced [34]. Total mRNA levels peak shortly $30 \mathrm{~min}$ after stimulation and sharply decrease thereafter. The translated Fos protein is part of AP-1 transcription factor complexes that bind enhancers at different gene promoters and contribute to a second wave of gene expression, which is responsible of cell fate commitment changes. Subsequently, posttranslational modifications target the Fos protein for degradation. This rapid and transient pattern of Fos expression is observed in a variety of cells, and under different stimuli. This fact provides compelling evidence that both up- and down-regulation of the fos gene are important to maintain cellular homeostasis.

Abundant information has been gathered on the regulation of gene promoter activity $[35,36]$. However, mechanisms regulating the decay of mature mRNAs are still poorly understood. Our work focused on the pathways affecting fos mRNA turnover. Particularly AUrich elements (ARE) present in the fos 3' UTR region have already been proved to affect the half-life of the fos mRNA. Fusing the fos 3' UTR into a stable reporter mRNAs induced decay but mRNA stability was regained when its ARE sequence was deleted [9]. Even though these regulatory elements are present in $8 \%$ of transcripts including a variety of early-responsive genes [8], the variable features of their sequences and the mechanisms by which they contribute to the stability of mRNAs bearing them is still mostly unknown. Identifying the biochemical pathways that are responsible to transduce the signal from membrane receptors to effector proteins that regulate mRNA decay events becomes then particularly important.

Studying fos expression in response to the activation of different MAPK pathways we found that only ERK1/2 signaling induced fos promoter activity. In a different approach, using pharmacological inhibitors we observed that, to our surprise, inhibiting the activation of the p38 MAPK increased the amount of fos mRNA, although p38 MAPK did not affect fos promoter activity. We therefore focused on the study of p38 MAPK dependent events regulating mRNA levels in the absence of potential promoter effects. Experiments to test the stability or instability of messengers in different cellular conditions tend to be designed using general transcription inhibitors (e.g. actinomycin D or $\alpha$-amanitin). These effective reagents are also strong stress inducers, and deleterious to the life of cells. Therefore, in order to avoid secondary effects when studying regulation of fos mRNA decay mediated by its 3' UTR we designed a vector tool that allows us to selectively control mRNA expression of a reporter. Our construct expresses a luciferase gene reporter in which we cloned the entire 3'UTR of fos downstream from the luciferase coding region. This vector allows for controlled transcription with a tetracycline responsive (TetOff) promoter. Using this reporter we avoid unnecessary secondary stress effects, as induction of any Stress Activated Protein Kinases (SAPKs), including p38 MAPK, or inhibition of de novo synthesis of participating factors. Transcripts produced by this reporter construct showed clearly that they are under an ARE-dependent mRNA decay control.

In accordance to the data obtained studying endogenous fos expression we found that inhibition of the p38 MAPK signaling pathway by using either dominant negative forms of the MEK3 dual specificity kinase (a well-known p38 MAPKK) or the p38 inhibitor SB203580, extended the half-life of the Luc-fos reporter mRNA. This effect may seem contradictory to previous reports in which p38 MAPK activation correlates with the stabilization of mRNAs. 
However these reports are based on different genes and cellular contexts. For instance, p38 MAPK activation in response to $\mathrm{Y}$-radiation and consequent HuR phosphorylation by this kinase was shown to be responsible for the stabilization of p21cip mRNA [24]. On the other hand p38 MAPK stabilizes cytokine and TNF- $\alpha$ mRNAs during an inflammatory response $[19,37,38]$. Particularly p38/MK2 signaling prevents TTP binding to target mRNAs and degradation extending the half-life of TNFa mRNA [39]. However the effect of p38 MAPK on mRNA stability does not need to be unique as p38-dependent and -independent effects, have already been reported [40]. It is accepted that the particular environment in which a given mRNA is transcribed and the identity and post-translational status of the RNA binding proteins present and involved can actually determine the fate of mRNAs. Our studies are focused on a normal, proliferating context, in which no stresses are applied.

HuR, KSRP and TTP are representative ARE binding proteins or AUBPs. By means of a LC-MS sequencing approach and using a biotinylated probe corresponding to the fos ARE sequence (40 bp long) that includes the 3 consensus sequences AUUUA we searched for associated proteins. Several experiments rendered similar results: AUBPs HuR and KSRP were isolated as major components of fos ARE binding complexes. This information was validated by Western blots using specific antibodies and by RNA Electromobility Shift Assays (EMSAs) performed with a radioactive probe containing fos ARE sequences and bacterially expressed recombinant proteins. While HuR was also detected in endogenous cell lysates by supershift using a specific antibody we found no evidence of TTP binding to the fos ARE by any of these approaches.

HuR stabilizing effects on mRNA stability have already been observed for several genes $[20,41,42]$ and in particular for fos [8]. Our experiments show that cotransfection of HuR expressing plasmids prevents decay of a reporter mRNA containing fos regulatory ARE regions. Accordingly, when we knocked down HuR expression by siRNAs we found a remarkable reduction on fos protein expression. An antagonistic role for HuR and KSRP in controlling ARE containing mRNA decay has already been reported for the stability of the iNOS mRNA [19]. Accordingly, we report here that overexpression of KSRP prevents fos reporter mRNA stabilization by HuR. In addition EMSAs performed using a fos ARE probe and increasing amounts of either recombinant protein (KSRP and HuR) showed that they form mutually exclusive complexes, similar to that reported for the iNOS ARE [25] (data not shown). The negative effect of KSRP on fos mRNA stability is also supported by a partial but significant reversion of the stabilizing effect of SB203580.

The notion that phosphorylation of HuR plays a crucial role in connecting extracellular signal inputs to a specific response is a complex subject of increasing interest [43]. We report here that, unlike the wild type version, a triple HuR mutant with relevant phosphorylation sites mutated to alanines (T118A, S202A, S221A) is unable to prevent decay of the reporter Luc-fos construct, highlighting the importance of functional phosphorylation sites in HuR. However and in contrast with reports obtained working under different conditions [24], we have found no evidence of direct phosphorylation of HuR by p38 MAPK. In addition, in our system, inhibition of p38 MAPK signaling significantly increases HuR phosphorylation both in solid-phase and streptag purification assays. Although counterintuitive, association of HuR with phosphatase $2 \mathrm{~A}$ inhibitors pp32 and APRIL has been described [33]. This interaction modulate HuR's ability to bind its target mRNAs in living cells and suggest a role in connecting HuR to pathways controlling the stability of ARE-containing mRNAs. Cotransfection of vectors expressing pp32 and APRIL significantly stabilized the Luc-fos reporter transcript. Accordingly incubation of cells with a pharmacological phosphatase inhibitor had a similar stabilizing effect. These results highlight the involvement of a phosphatase activity in the promotion of fos mRNA decay. 
HuR binding to specific sequences is necessary to stabilize target mRNAs. As shown by our own data and the literature HuR phosphorylation might have a positive contribution to this effect $[22,24]$. Using a radioactive probe designed with fos ARE sequences we performed RNA EMSAs that revealed the assembly of a complex (C2) that contains HuR as an important component according to mobility supershift assays. Time course experiments after the addition of PDGF showed a peak of intensity in the formation of C2 complexes that is remarkably coincident in time with a peak in fos mRNA expression. Interestingly, C2 complexes remained assembled longer upon incubation of cells with a p38 MAPK pharmacological inhibitor. Taken together these results and the phosphorylation assays, we can suggest a role for p38 MAPK in HuR dephosphorylation and consequent disassembling of HuR/fos mRNA complexes.

Summarizing, our results are consistent with the following sequence of events as depicted in Figure 8. Upon growth factor stimulation of resting cells a variety of MAPK pathways are activated showing two distinctive temporal patterns as shown in Table 1. While "Early Activated MAPKs" (such as ERK2 and 5) attain maximal activity at $5 \mathrm{~min}$ after stimulation, "Late Activated MAPKs" (JNKs and p38s) display activity at later times and sometimes for longer intervals. Early activation of ERK2 is consistent with its role as activator of the fos promoter. Once translated, Fos protein is phosphorylated by different MAPKs $[1,4]$ and as a member of AP-1 transcription factors conveys signals further to other gene promoters. By the time enough Fos protein has been produced and activated, fos mRNA might be no longer needed and p38 MAPKs promote its decay. Phosphorylated HuR prevents fos mRNA decay, and might become later the subject of p38 MAPK dependent dephosphorylation. This event might involve the release of phosphatase inhibitors and facilitate changes in the repertoire of proteins bound to the fos ARE. Lower amounts of HuR bound to the fos mRNA might therefore allow for the assembly of a protein complex of different composition at the ARE sequences, most probably including KSRP, and targeting the fos mRNA molecules to degradation. This AUBP exchange at regulatory sites in target mRNAs has already been described for other target mRNAs [25,44]. The idea of a phosphorylation-regulated AUBP exchange [45] presents an interesting model for switching between situations that promote conditions of stability or instability for specific mRNAs and contribute to gene expression regulation.

In general, the role of $\mathrm{p} 38 \mathrm{MAPK}$ as a mediator of mRNA stability seems to be highly dependent upon the gene model under study and the environmental context of the cellular system. Most reports pointing p38 MAPK as a stabilizing mRNA pathway describe genes involved in inflammatory responses under stress conditions of myogenic differentiation, while our data show a destabilizing role for p38 MAPK on fos mRNA, in the context of proliferative stimulation. Ultimately, as for promoter activity, extracellular originated signaling regulates gene expression at the mRNA decay level through a variety of molecular components. The final outcome is of fascinating complexity. Our results show how concerted early (ERK) or late (p38 and JNK) MAPK activation can contribute to both rapid and transient activation of the early responsive gene fos. Our working model provides a blueprint for the events taking place, identifying additional components of the complex assembled at the fos ARE (including proteins and miRNAs). Its precise regulation by p38 MAPK, including the activation of a putative HuR phosphatase, constitute current challenges that warrant further investigation. 


\section{AUTHOR CONTRIBUTION}

Maria Degese, Tamara Tanos and Omar Coso conceived and designed the experiments. Maria Degese, Tamara Tanos and Diego Chiappe performed experiments. Maria Degese, Tamara Tanos, Pablo Echeverria, Jonathan LaMarre, Silvio Gutkind and Omar Coso analyzed the data. Maria Degese, Tamara Tanos, Julian Naipauer, Tim Gingerich, Jonathan LaMarre, Silvio Gutkind and Omar Coso helped set-up techniques and/or discussed preliminary ideas and data. Tamara Tanos, Julian Naipauer, Diego Chiappe, Tim Gingerich, Jonathan LaMarre and Silvio Gutkind contributed reagents, materials and analysis tools. Pablo Echeverria led the bioinformatics search. Maria Degese and Omar Coso wrote the paper.

\section{ACKNOWLEDGEMENTS}

We want to thank all members of the IFIBYNE - CONICET and Departamento de Química Biológica FCEN - UBA for sharing equipment and for their support and thoughtful advice. We thank Dr. Didier Picard and his staff (UNIGE-Geneva, Switzerland) for their useful suggestions. We thank Dr. Joan Steitz (Yale-HHMI, USA) for providing the APRIL, pp32 and SET constructs, Dr. Gary Brewer (Rutgers-RWJMS, USA) for the AUF1 vectors, Dr. Angel Nebreda (IRBBarcelona, Spain) for the human cDNA of WT and mutated versions of HuR and Dr. Hartmut Kleinert (Uni-Mainz, Germany) for the KSRP constructs. The authors thank Daniela Capiatti (INGEBI-UBA, Argentina) for the PP2A inhibitor Endothall.

\section{FUNDING}

This work was supported by the University of Buenos Aires - UBA, Consejo Nacional de Ciencia y Tecnica - CONICET and Agencia Nacional de Promocion Cientifica y Tecnologica - ANPCyT, Argentina. We received additional support from the Union for International Cancer Control UICC with 2 ICRETT Travel Fellowships [ICR-08-120] and [ICR-11-008]. 


\section{Legends to the figures}

Figure 1: Analysis of fos expression as a response to mitogenic stimuli. MAPKs involvement in fos promoter activity.

(A) NIH3T3 cells expressing the M1 muscarinic receptor (m1.2 cells) were stimulated with the agonist Carbachol (Ch) to a final concentration of $100 \mathrm{mM}$ following a time course. RNA was extracted in order to perform a northern blot assay using a specific fos probe. (B) Real time qPCR of fos mRNA in m1.2 stimulated with Ch (100 mM, left graph) and NIH3T3 incubated in the presence of PDGF (100ng/mL, right graph), levels of GAPDH expression were used to normalize. (C) Luciferase assays. pc-fosLuc (pGL3-c-fos promoter-Luciferase) was cotransfected with vectors expressing different MAPKs and their activating MAPKKs, in NIH3T3. (D) Similar to (C) stimulating MAPKs in m1.2 cells with indicated agonists (Ch, left graph or PDGF, right graph).

Figure 2: MAPK involvement on fos mRNA expression.

(A) Northern blot. Endogenous fos expression was analyzed in mRNA extracts of m1.2 cells pretreated with MAPKs inhibitors (MEK inhibitor, PD98059 $20 \mu \mathrm{M}$ and p38 inhibitor, SB203580 $10 \mu \mathrm{M})$ stimulated with Ch for 40 minutes. (B) Same experiment as in (A), but in this case fos mRNA was quantified by qPCR. m1.2 cells stimulated with $\mathrm{Ch}$, left panel and NIH3T3 cells stimulated with PDGF, right panel. *: $p$-value $<0.05 ;{ }^{* *}$ : $p$-value $<0.01 ;{ }^{* * *}$ : $p$-value $<0.001$.

Figure 3: Role of the p38 MAPK pathway in regulating levels of fos mRNA expression.

(A) Schematic representation of fos mRNA structure. An AU-rich region known as ARE is highlighted. (B) The entire murine 3'UTR of fos in its wild type (WT) version was inserted in the pTRE2hygLuc vector (Luc-fos); this plasmid has a tetracycline responsive promoter (also responsive to analogs such as doxycycline) that drives transcription only in the absence of antibiotic. The Luc-fos $\triangle A R E$ reporter is identical to the WT except for a 69 bp deletion encompassing the ARE region. Luc $\beta G$ lobin has the entire 3'UTR region of the $\beta$-globin gene cloned in pTRE2hygLuc and contains no ARE regulatory elements. (C) HeLa TetOff cells were

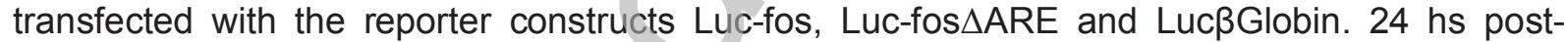
transfection cells were incubated with tetracycline (1ug/ul final concentration) for a maximum period of $5 \mathrm{hrs}$, RNA was extracted and a RT-qPCR was performed to quantitate luciferase total mRNA. (D) NIH3T3 TetOff cells were cotransfected with the reporters Luc-fos and LucßGlobin and vectors expressing either the dominant negative (MEK3AA) or constitutively activated (MEK3EE) versions of the MAPKK MEK3, a p38 regulator. 24 hrs post-transfection cells were incubated with doxycycline and processed as in (C). (E) Same experiments as in (C) but pretreating cells with kinase inhibitors for p38 MAPK (SB203580, 10 uM final) and MK2 (MK2ai, 50uM) before tetracycline addition. (F) Proliferating NIH3T3 cells were starved for $12 \mathrm{hrs}$ and stimulated with PDGF following a time course, in the absence or presence of kinase inhibitors SB203580 and MK2ai. RT-qPCR of endogenous fos mRNA (normalized with GAPDH) was performed. The ratio of fos mRNA present at 60 minutes to mRNA present at 30 minutes is shown. *: $p$-value $<0.05 ;{ }^{* *}$ : $p$-value $<0.01 ;{ }^{* * *}$ : $p$-value $<0.001$.

Figure 4: AUBP proteins binding to the fos ARE sequence.

(A) Biotinylated probes of fos mRNA ARE region (either WT or mutated as described in Experimental) were incubated with HEK293 cell lysates stimulated with EGF $(10 \mathrm{ng} / \mathrm{mL})$ and then purified with streptavidin-agarose. Silver stained PAGE SDS gel (upper panel) is shown. Western blot of these purified complexes (lower panel) identified HuR and KSRP binding differentially to the WT probe. (B) Network-based analysis of p38 MAPK and AUBP interactors. PPIs for the query proteins were retrieved and organized in maps together with literature 
curation data and functional metadata using the software Cytoscape [30]. Nodes (proteins) are connected by edges (lines) representing their known physical interactions. In grey are showed those proteins also found in the LC-MS sequentiation experiments.

Figure 5: Effect of different relevant factors on fos mRNA decay.

(A) HeLa TetOff cells were transfected with the reporter constructs Luc-fos, Luc-fos $\triangle A R E$ and Luc $\beta$ Globin along with vectors that were either empty or expressed murine HA tagged HuR. After addition of tetracycline, mRNA decay assays were run. The western blot on the right panel shows expression of murin wild type HA-HuR. (B) siRNA depletion of HuR in NIH3T3 cells transfected with HuR siRNA or scramble siRNA. Starved cells were stimulated with PDGF for 30 minutes, after that period cells were lysed and levels of expression of endogenous HuR and Fos proteins were visualized in western blots. (C) A mRNA decay assay was run on extracts from cells transfected with either empty plasmid or expressing wild type or a triple mutant of human HuR (T118A, S202A, S221A). The panel on the right validates expression as seen on a western blot. (D) As in (C) but cotransfecting the reporter with vectors that express the FLAG tagged PP2A inhibitors and HuR binding proteins, pp32 and APRIL. (E) As in (C) but in cells transfected with plasmids expressing EGFP-KSRP. Treatment with SB203580 was performed as indicated in the upper panel. In the middle panel cells were cotransfect with the reporter and also with vectors that express EGFP-KSRP or HA-HuR. A western blot against GFP is shown on the lower panel. (F) Endothall, a PP2A and PP1 inhibitor was also tested at $50 \mu \mathrm{M}$ final concentration. HeLa TetOff cells transfected with the Luc-fos reporter were incubated with Endothall and/or tetracycline for different times as indicated and mRNA present in the extract was tested by RT - qPCR. In western blots NT correspond to non-transfected cells. *: p-value $<0.05 ;{ }^{* *}$ : $p$-value $<0.01 ;{ }^{* * *}$ : $p$-value $<0.001$.

Figure 6: Determination of HuR phosphorylation state.

(A) Solid phase assay. Proliferating NIH3T3 were starved for $12 \mathrm{hrs}$ and stimulated with PDGF for the indicated times. Cell lysates were incubated overnight with bacterially expressed recombinant GST-HuR coupled with glutathione sepharose beads. Protein complexes bound to the beads were washed, incubated in the presence of radioactive $\left[\mathrm{Y}^{32} \mathrm{P}\right]-\mathrm{ATP}$ and this reaction mix was run in a PAGE- SDS gel that was dried and autoradiographed. Lysates from cells treated with anisomycin or vehicle were tested for GST-ATF2 and GST phosphorylation activity, as positive and negative controls, respectively. (B) Statistical analysis of 3 independent solid phase assays as in A. (C) HEK293 cells were transfected with a vector expressing Strep2 tagged murine HuR, stimulated with EGF and inclubated with Streptactin-agarose beads. Pulled down proteins were run on PAGE-SDS gels and blotted with anti-phospho-Serine antibodies. western blot using anti-StrepTag show the total amount of HuR. The right panel corresponds to cells pretreated with SB203580 and the left panel untreated cells. ${ }^{*}$ : p-value $<0.05$; ${ }^{* *}$ : $p$ value $<0.01 ;{ }^{* * *}$ : $p$-value $<0.001$.

Figure 7: Protein complexes binding to fos ARE.

(A) A radioactive RNA probe containing the fos ARE sequence was incubated with NIH3T3 cell protein lysates, run on a non-denaturing gel and specific complex formation was studied by autoradiography. Increasing amount of unlabeled probes containing the fos ARE or $\beta$-globin sequences were added as specific and non-specific controls respectively. An additional control was performed using proteinase $\mathrm{K}(\mathrm{PK})$. The two major complexes studied are showed with arrows as $\mathrm{C} 1$ (complex1) and C2 (complex2). (B) A similar experiment was performed using bacterially expressed recombinant GST (HuR, TTP and BRF1) or 6xHis fusion proteins (KSRP) incubated with the ARE probe. (C) Presence of putative protein candidates in the complexes assembled at ARE sequences was tested by incubating cell lysates with the probe in the presence of antibodies against representative AUBP family members. A supershift is indicated 
by an arrow. (D) The same experiment was performed using lysates of NIH3T3 cells that were stimulated with PDGF over a time course. The intensity of two major complexes (C2 containing (HuR) or not, C1) was quantitated and depicted in the graph. (E) Intensity of the HuR containing complexes was tested in lysates from cells stimulated with PDGF after preincubation with the p38 MAPK inhibitor SB203580 or vehicle. ${ }^{*}$ : $p$-value $<0.05$; ${ }^{* *}$ : $p$-value $<0.01 ;{ }^{* * *}$ : $p$-value $<0.001$.

Figure 8: Concerted role for early and late activated MAPKs in the rapid and transient expresión of the fos early responsive gene.

Our current view of the model shows that while early activated MAPKs (ERK2) trigger fos promoter induction, late activated MAPKs (p38) regulate mRNA decay. After mitogenic receptor stimulation, once a time interval for peak Fos protein translation and phosphorylation has been completed, low levels of fos mRNA are restored, through phosphorylation dependent events. The assembly of a protein complex of different composition at the ARE sequence, probably including KSRP, targets the fos mRNA to degradation. 


\section{Reference List}

1. Tanos, T., Marinissen, M. J., Coluccio-Leskow, F., Hochbaum, D., Martinetto, H., Gutkind, J. S., and Coso, O. A. (2005) Phosphorylation of c-Fos by members of the p38 MAPK family. Role in the AP-1 response to UV light. J.Biol.Chem. 280, 18842-18852.

2. Cochran, B. H., Zullo, J., Verma, I. M., and Stiles, C. D. (1984) Expression of the c-fos gene and of an fos-related gene is stimulated by platelet-derived growth factor. Science 226, 1080-1082.

3. Coso, O. A., Chiarello, M., Kalinec, G., Kyriakis, J. M., Woodgett, J., and Gutkind, J. S. (1995) Transforming $G$ protein-coupled receptors potently activate JNK (SAPK). Evidence for a divergence from the tyrosine kinase signaling pathway. J.Biol.Chem. 270, 5620-5624.

4. Monje, P., Marinissen, M. J., and Gutkind, J. S. (2003) Phosphorylation of the carboxylterminal transactivation domain of $\mathrm{c}$-Fos by extracellular signal-regulated kinase mediates the transcriptional activation of AP-1 and cellular transformation induced by platelet-derived growth factor. Mol.Cell.Biol. 23, 7030-7043.

5. Yang, SH., Sharrocks, A. D., and Whitmarsh, A. J. (2003) Transcriptional regulation by the MAP kinase signaling cascades. Gene 320, 3-21.

6. O'Donnell, A., Odrowaz, Z., and Sharrocks, A. D. (2012) Immediate-early gene activation by the MAPK pathways: what do and don't we know? Biochem.Soc. Trans. 40, 58-66.

7. Gingerich, T. J., Feige, J.J., and LaMarre, J. (2004) AU-rich elements and the control of gene expression through regulated mRNA stability. Anim. Health Res.Rev. 5, 49-63.

8. Barreau, C., Paillard, L., and Osborne, B. (2006) AU-rich elements and associated factors: are there unifying principles? Nucleic Acids Res. 33, 7138-7150.

9. Shyu, A. B., Greenberg, M. E., and Belasco, J. G. (1989) The c-fos transcript is targeted for rapid decay by two distinct mRNA degradation pathways. Genes Dev. 3, 60-72.

10. Chen, C. A., Xu, N., and Shyu, A. B. (1995) mRNA decay mediated by two distinct AUrich elements from c-fos and granulocyte-macrophage colony-stimulating factor transcripts: different deadenylation kinetics and uncoupling from translation. Mol.Cell.Biol. 15, 5777-5788.

11. Ma, WJ., Cheng, S., Campbell, C., Wright, A., and Furneaux, H. (1996) Cloning and characterization of HuR, a ubiquitously expressed Elav-like protein. J.Biol.Chem. 271, 8144-8151.

12. Kajita, Y., Nakayama, J., Aizawa, M., and Ishikawa, F. (1995) The UUAG-specific RNA binding protein, heterogeneous nuclear ribonucleoprotein D0. Common modular structure and binding properties of the 2xRBD-Gly family. J.Biol.Chem. 270, 2216722175.

13. Chou, CF., Mulky, A., Maitra, S., Lin, WJ., Gherzi, R., Kappes, J., and Chen, CY. (2006) Tethering KSRP, a decay-promoting AU-rich element-binding protein, to mRNAs elicits mRNA decay. Mol.Cell.Biol. 26, 3695-3706.

14. Carballo, E., Lai, W. S., and Blackshear, P. J. (1998) Feedback inhibition of macrophage tumor necrosis factor-alpha production by tristetraprolin. Science 281, 1001-1005.

15. Stoecklin, G., Colombi, M., Reineri, I., Leuenberger, S. A., Mallaun, M., Schmidlin, M., Gross, B., Lu, M., Kitamura, T., and Moroni, C. (2002) Functional cloning of BRF1, a regulator of ARE-dependent mRNA turnover. EMBO J. 21, 4709-4718.

16. Chen, CY., Gherzi, R., Ong, SE., Chan, E. L., Raijmakers, R., Prujin, G. J. M., Stoecklin, G., Moroni, C., Mann, M., and Karin, M. (2001) AU binding proteins recruit the exosome to degrade ARE-containing mRNAs. Cell 107, 451-464. 
17. Zhu, W., Brauchle, M. A., Di Padova, F., Gram, H., New, L., Ono, K., Downey, J. S., and Han, J. (2001) Gene suppression by tristetraprolin and release by the p38 pathway. Am.J.Physiol.Lung Cell.Mol.Physiol. 281, 499-508.

18. Stoecklin, G., Stubbs, T., Kedersha, N., Wax, S., Rigby, W. F. C., Blackwell, T. K., and Anderson, P. (2004) MK2-induced tristetraprolin:14-3-3 complexes prevent stress granule association and ARE-mRNA decay. EMBO J. 23, 1313-1324.

19. Mahtani, K. R., Brook, M., Dean, J. L. E., Sully, G., Saklatvala, J., and Clark, A. R. (2001) Mitogen-activated protein kinase p38 controls the expression and posttranslational modification of tristetraprolin, a regulator of tumor necrosis factor alpha mRNA stability. Mol.Cell.Biol. 21, 6461-6469.

20. Chen, C. A., Xu, N., and Shyu, A. B. (2002) Highly selective actions of HuR in antagonizing AU-rich element-mediated mRNA destabilization. Mol.Cell.Biol. 22, 72687278 .

21. Abdelmohsen, K., Pullman, R., Lal, A., Kim, H. H., Galban, S., Yang, X., Beltrow, J. D., Walker, M., Shubert, J., Gillespie, D. A., Furneaux, H., and Gorospe, M. (2007) Phosphorylation of HuR by Chk2 regulates SIRT1 expression. Mol.Cell 25, 543-557.

22. Kim, H. H., Abdelmohsen, K., Lal, A., Pullman, R., Yang, X., Galban, S., Srikantan, S., Martindale, J. L., Blethrow, J., Shokat, K. M., and Gorospe, M. (2008) Nuclear HuR accumulation through phosphorylation by Cdk1. Genes Dev. 22, 1804-1815.

23. Doller, A., Winkler, C., Azrilian, I., Schulz, S., Hartman, S., Pfeilsschifter, J., and Eberhardt, W. (2011) High-constitutive HuR phosphorylation at Ser 318 by PKC\{delta\} propagates tumor relevant functions in colon carcinoma cells. Carcinogenesis 32, 676685.

24. Lafarga, V., Cuadrado, A., Lopez de Silanes, I., Bengochea, R., Fernandez-Capetillo, O., and Nebreda, A. R. (2009) p38 Mitogen-activated protein kinase- and HuRdependent stabilization of p21(Cip1) mRNA mediates the $G(1) / S$ checkpoint. Mol.Cell.Biol. 29, 4341-4351.

25. Linker, K., Pautz, A., Fechir, M., Hubrich, T., Greeve, J., and Kleinert, H. (2005) Involvement of KSRP in the post-transcriptional regulation of human iNOS expressioncomplex interplay of KSRP with TTP and HuR.Nucleic Acids Res. 33, 4813-4827.

26. Gallouzi, I., Brennan, C. M., and Steitz, J. A. (2001) Protein ligands mediate the CRM1dependent export of HuR in response to heat shock. RNA 7, 1348-1361.

27. Naipauer, J., Gatelli, A., Degese, M., Slomiansky, V., Werthmeier, E., LaMarre, J., Castilla, L., Abba, M., Kordon, E., and Coso, O. A. (2013) The use of alternative polyadenylation sites renders integrin $\beta 1$ (Itgb1) mRNA isoforms with differential stability during mammary gland development. Biochem.J. 454, 345-357.

28. You, Y., Chen, C A., and Shyu, A. B. (1992) Two cellular proteins bind specifically to a purine-rich sequence necessary for the destabilization function of a c-fos protein-coding region determinant of mRNA instability. Mol.Cell.Biol. 12, 2931-2940.

29. Echeverria, P. C., Forafonov, F., Pandey, D. P., Mulebach, G., and Picard, D. (2011) Detection of changes in gene regulatory patterns, elicited by perturbations of the Hsp90 molecular chaperone complex, by visualizing multiple experiments with an animation. BioData Mining 10.1186/1756-0381 4-15.

30. Cline, M., Smoot M, Cerami E, Kuchinsky A, Landys N, Workman C, Christmas R, AvilaCampilo I, Creech M, Gross B, Hanspers K, Isserlin R, Kelley R, Killcoyne S, Lotia S, Maere S, Morris J, Ono K, Pavlovic V, Pico AR, Vailaya A, Wang PL, Adler A, Conklin BR, Hood L, Kuiper M, Sander C, Schmulevich I, Schwikowski B, Warner GJ, Ideker T, Bader GD. (2007) Integration of biological networks and gene expression data using Cytoscape. Nat.Protoc. 2, 2366-2382. 
31. Echeverria, P. C., Bernthaler, A., Dupuis, P., Mayer, B., and Picard, D. (2011) An interaction network predicted from public data as a discovery tool: application to the Hsp90 molecular chaperone machine. PLoS One 6 (10), 26044.

32. Briata, P., Forcales SP., Ponassi, M., Corte, G., Chen, CY., Karin, M., Puri, PL. and Gherzi, R. (2005) p38-dependent phosphorylation of the mRNA decay-promoting factor KSRP controls the stability of select myogenic transcripts. Mol. Cell 20, 891-903.

33. Brennan, C. M., Gallouzi, I., and Steitz, J. A. (2000) Protein ligands to HuR modulate its interaction with target mRNAs in vivo. J.Cell Biol. 151, 1-13.

34. Fromm, C., Coso, O. A., Montaner, S., Xu, N., and Gutkind, J. S. (1997) The small GTPbinding protein Rho links $G$ protein-coupled receptors and Galpha12 to the serum response element and to cellular transformation. PNAS 94, 10098-10103.

35. Spitz, F. and Furlong, E. (2012) Transcription factors: from enhancer binding to developmental control. Nat.Rev.Genet. 13, 613-626.

36. Johnson, D. and Dent, S. (2013) Chromatin: receiver and quarterback for cellular signals. Cell 152, 685-689.

37. Otkjaer, K., Holtman, H., Kragstrup, T. W., Paludan, S. R., Johansen, C., Gaesthel, M., Kragballe, K., and Iversen, L. (2010) The p38 MAPK regulates IL-24 expression by stabilization of the 3' UTR of IL-24 mRNA. PLoS One 5 (1), 8671.

38. Winzen, R., Kracht, M., Ritter, B., Wilhem, A., Chen, C.A., Shyu, A. B., Muller, M., Gaesthel, M., Resch, K., and Holtmann, H. (1999) The p38 MAP kinase pathway signals for cytokine-induced mRNA stabilization via MAP kinase-activated protein kinase 2 and an AU-rich region-targeted mechanism. EMBO J. 18, 4969-4980.

39. Hitti, E., lakovleva, T., Brook, M., Deppenmeier, S., Gruber, A. D., Radzioch, D., Clark, A. R., Blackshear, P. J., Kotlyarov, A., and Gaesthel, M. (2006) Mitogen-activated protein kinase-activated protein kinase 2 regulates tumor necrosis factor mRNA stability and translation mainly by altering tristetraprolin expression, stability, and binding to adenine/uridine-rich element. Mol.Cell.Biol. 26, 2399-2407.

40. Frevel, M. A. E., Bakheet, T., Silva, A. M., Hissong, J. G., Khabar, K. S. A., and Williams, B. R. G. (2003) p38 Mitogen-activated protein kinase-dependent and -independent signaling of mRNA stability of AU-rich element-containing transcripts. Mol.Cell.Biol. 23, 425-436.

41. Fan, X. C. and Steitz, J. A. (1998) Overexpression of HuR, a nuclear-cytoplasmic shuttling protein, increases the in vivo stability of ARE-containing mRNAs. EMBO J. 17, 3448-3460.

42. Lopez de Silanes, I., Lal, A., and Gorospe, M. (2005) HuR: post-transcriptional paths to malignancy. RNA biol. 2, 11-13.

43. Eberhardt, W., Doller, A., and Pfeilsschifter, J. (2012) Regulation of the mRNA-binding protein HuR by posttranslational modification: spotlight on phosphorylation Curr.Protein Pept.Sci. 13, 380-390.

44. Doller, A., Schlepckow, K., Schwalbe, H., Pfeilsschifter, J., and Eberhardt, W. (2010) Tandem phosphorylation of serines 221 and 318 by protein kinase Cdelta coordinates mRNA binding and nucleocytoplasmic shuttling of HuR. Mol.Cell.Biol. 30, 1397-1410.

45. Tiedje,C.,Ronkina,N., Tehrani,M., Dhamija,S., Laass,K., Holtmann,H., Kotlyarov,A., Gaesthel,M. (2012) The p38/MK2-driven exchange between tristetraprolin and HuR regulates AU-rich element-dependent translation. PLoS Genet. 8 (9), 1002977. 
Figure 1

A

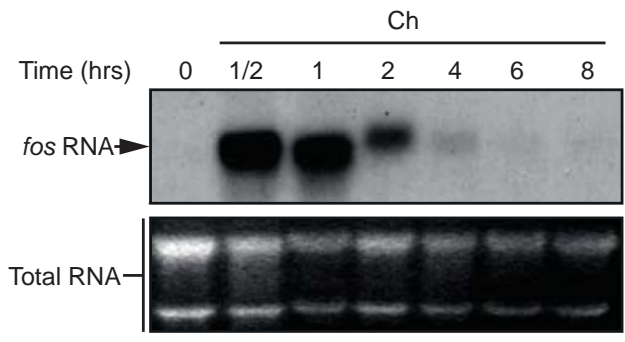

B
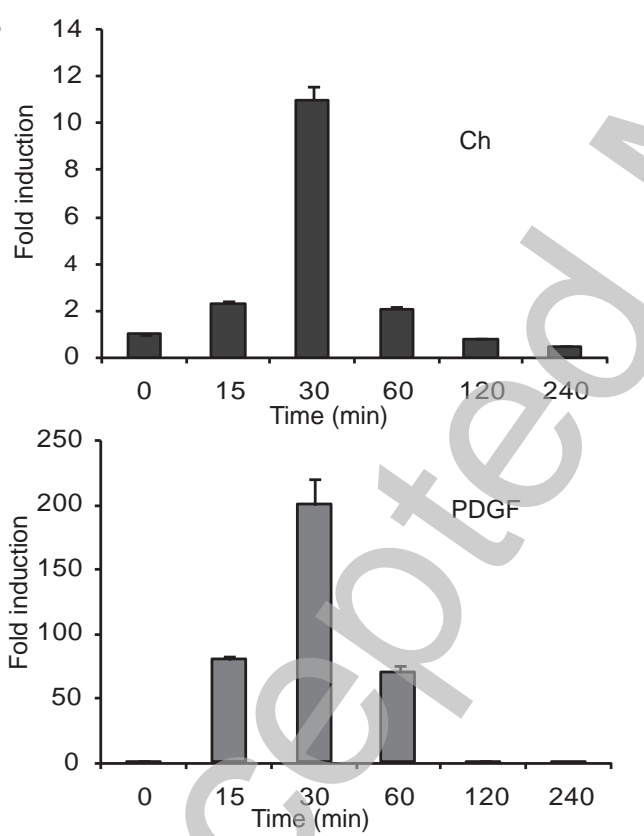

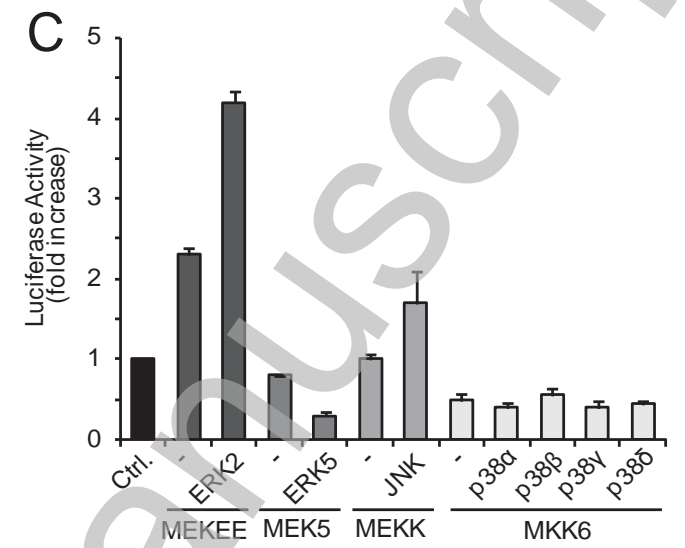

D ${ }^{14}$
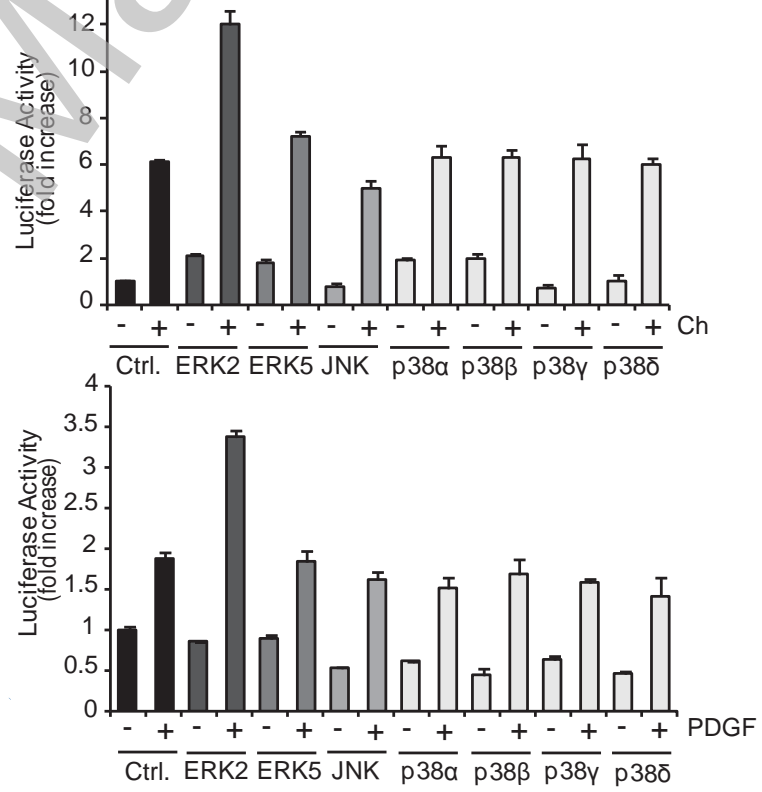
Figure 2
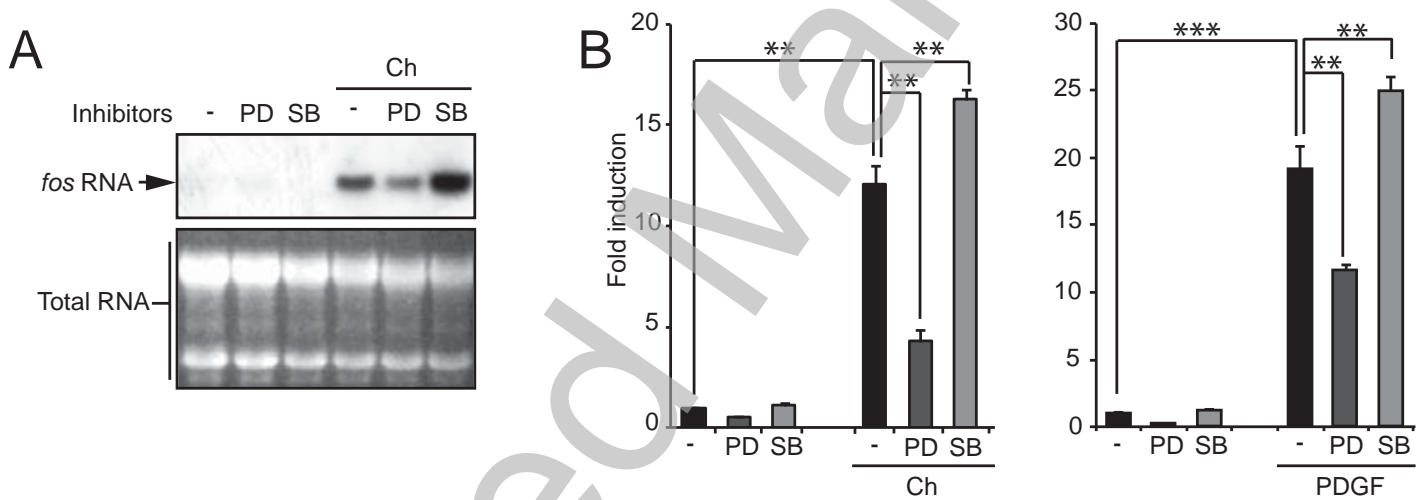
Figure 3

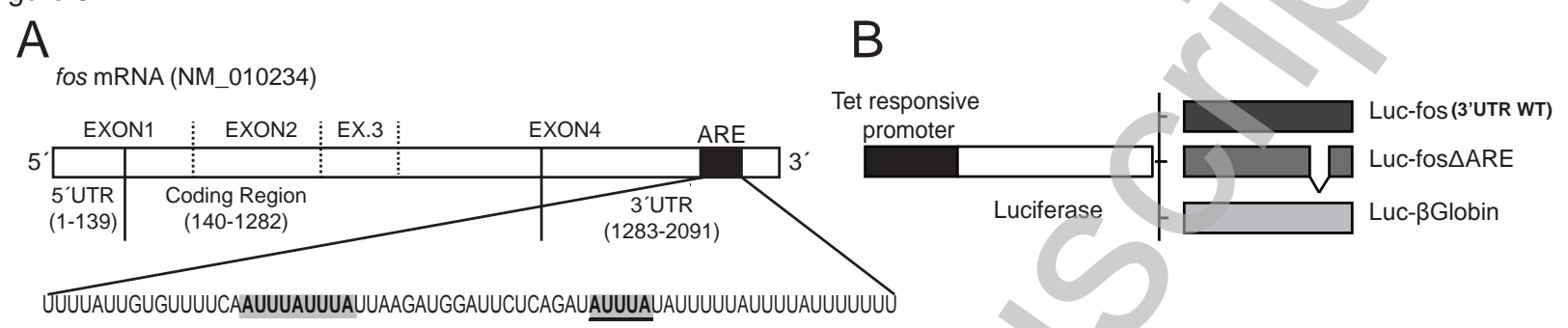

C

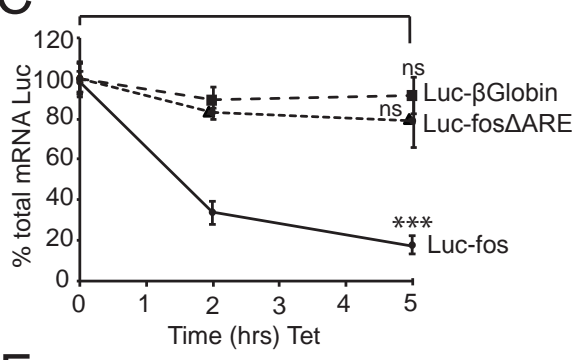

D

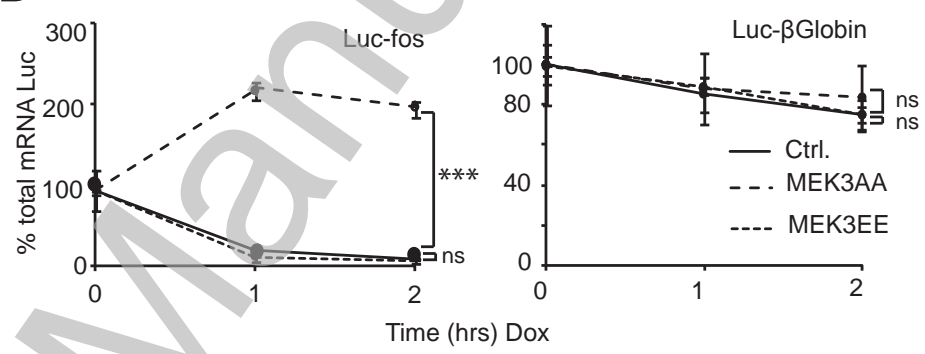

Time (hrs) Dox

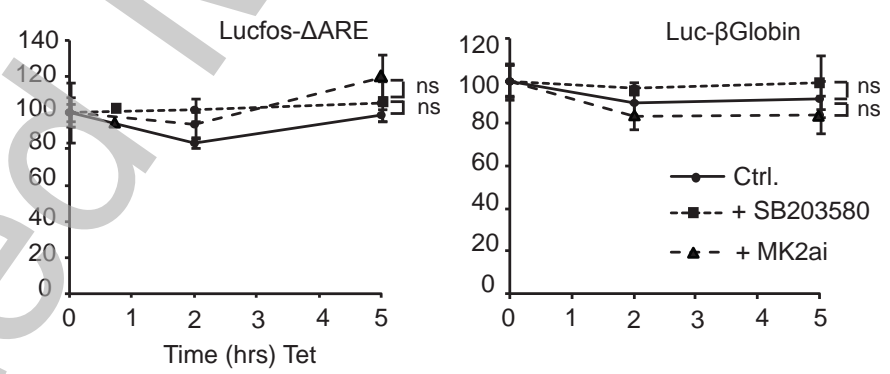

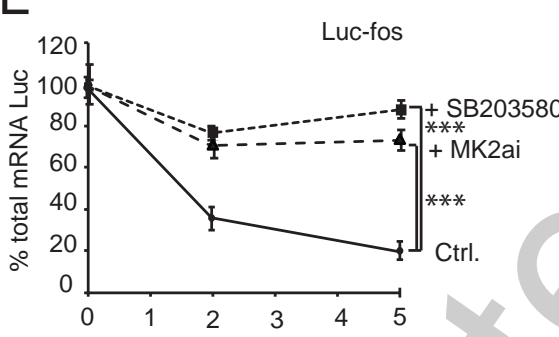

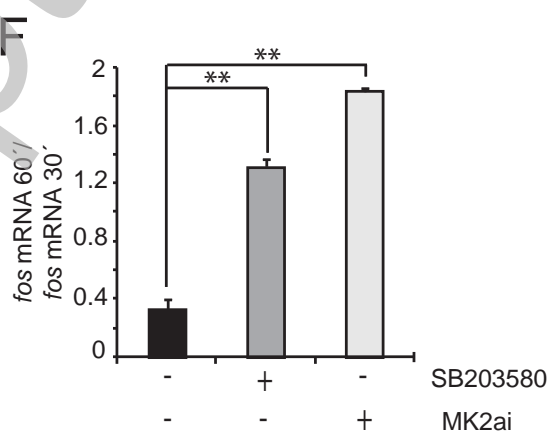

Licenced copy. Copying is not permitted, except with prior permission and as allowed by law. (C) 2015 The Authors Journal compilation (C) 2015 Biochemical Society 
Figure 4

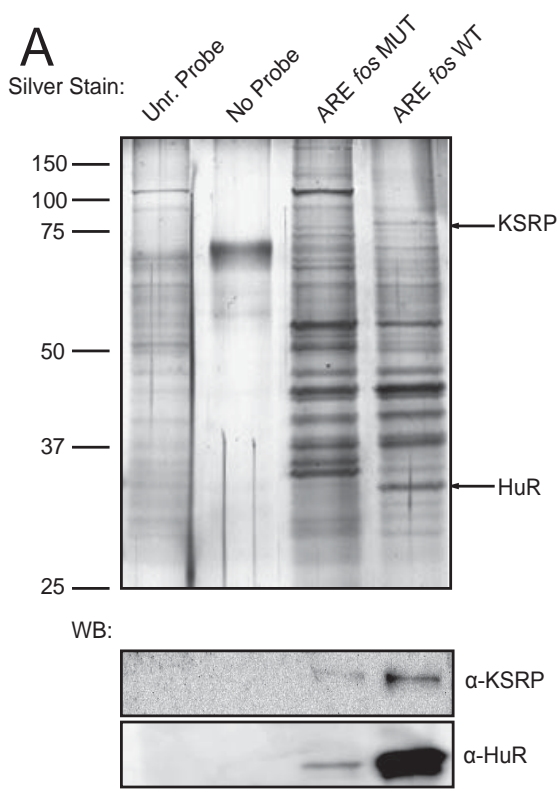

B

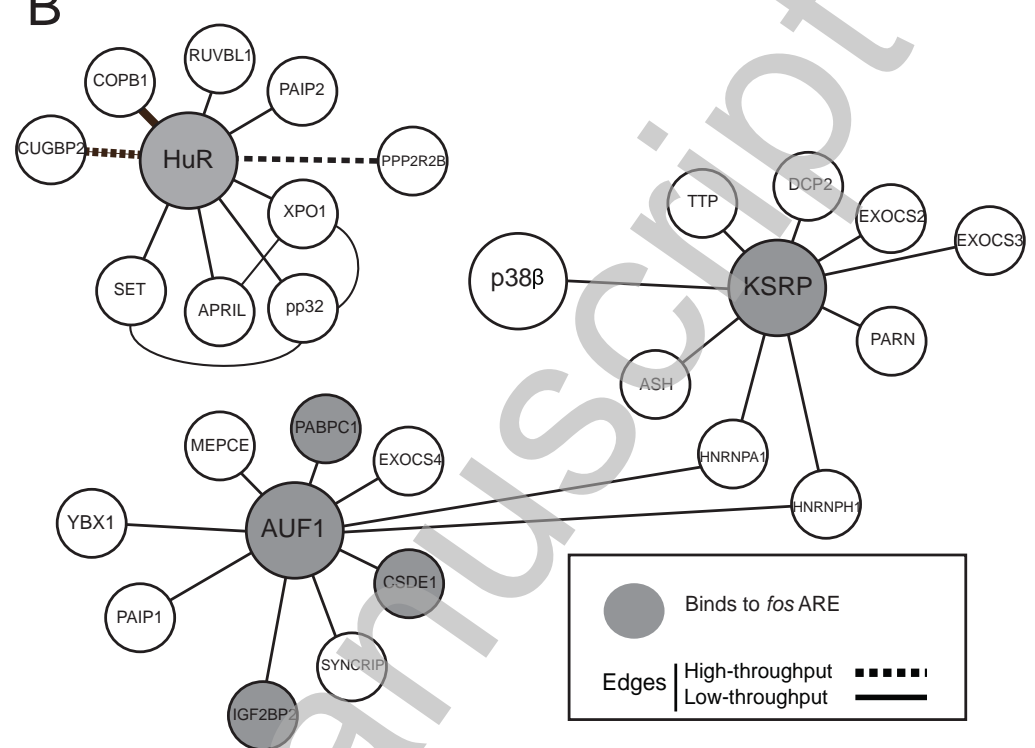


Figure 5

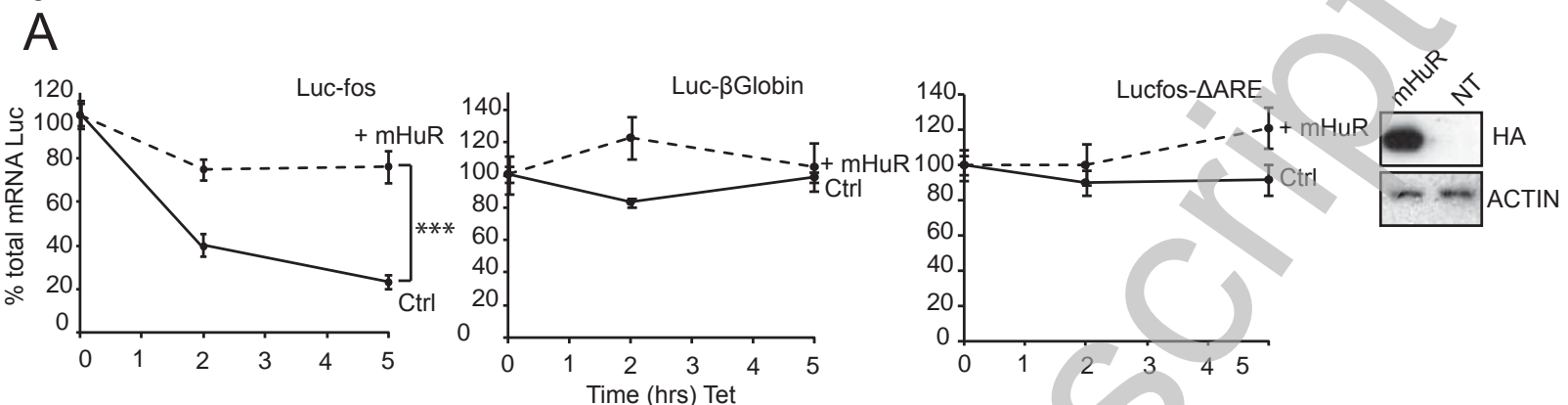

B

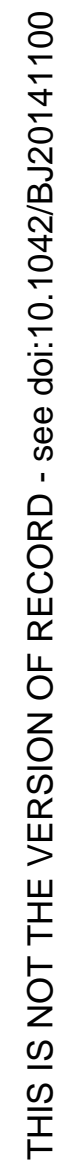
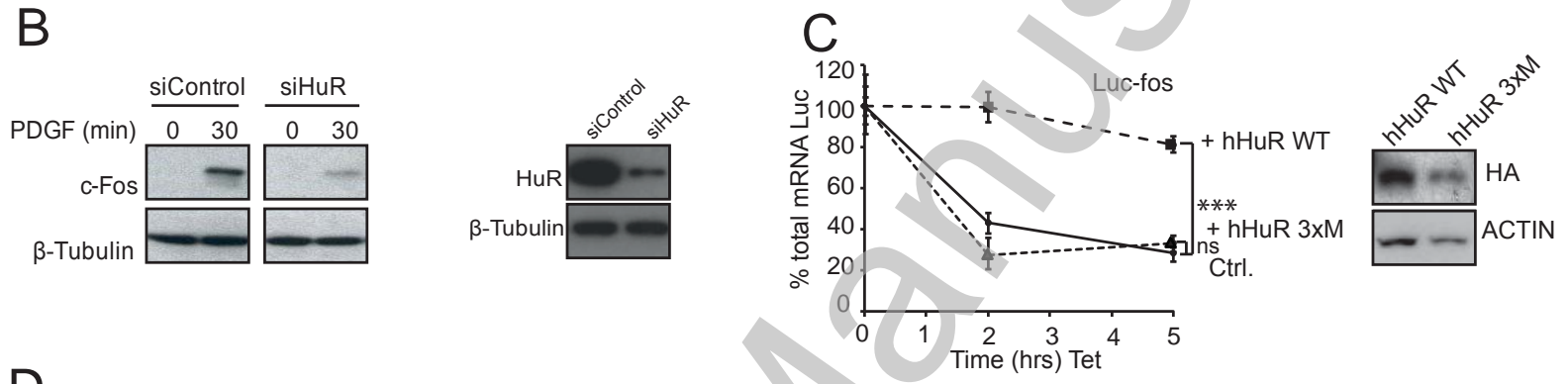

D
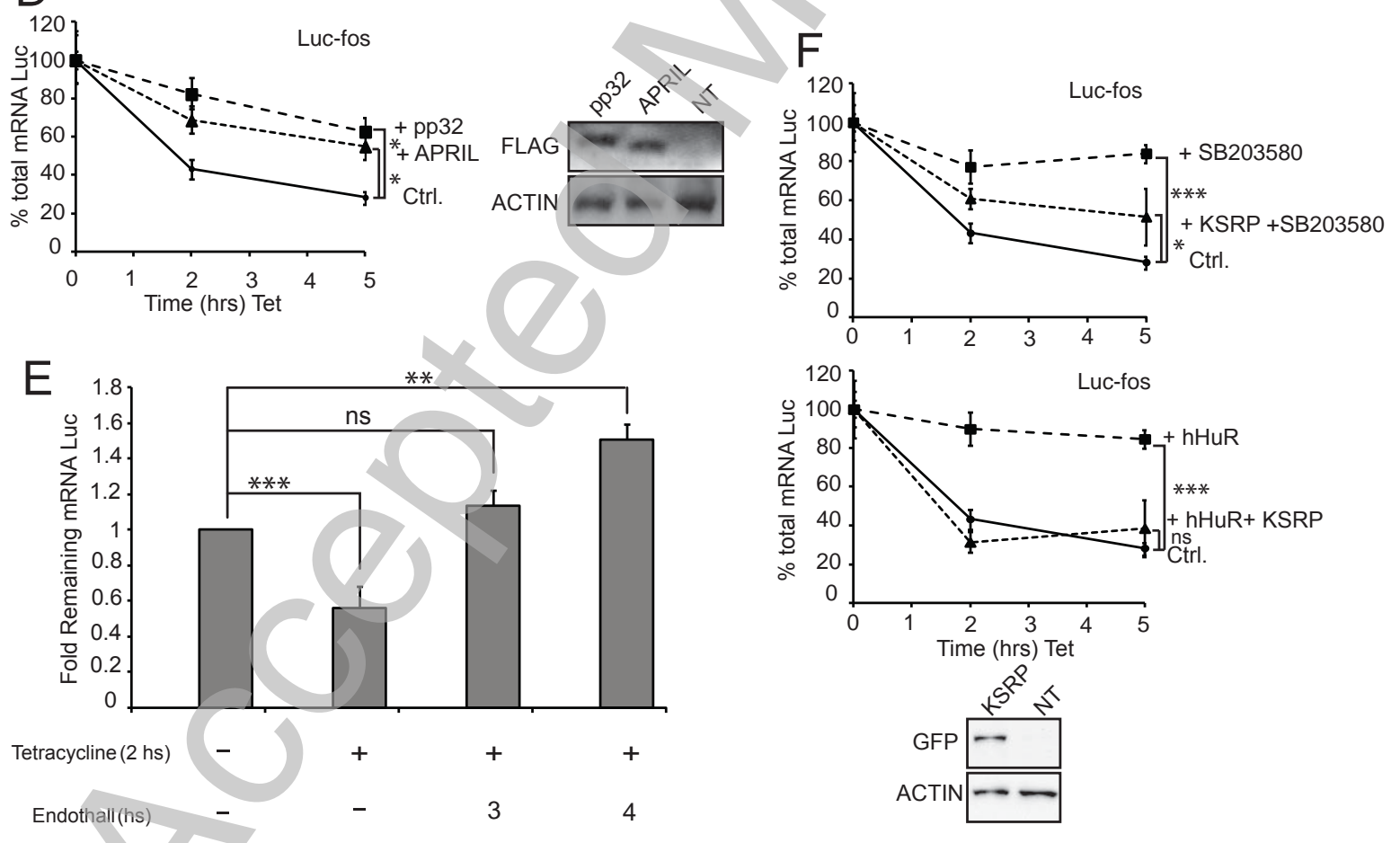
Figure 6

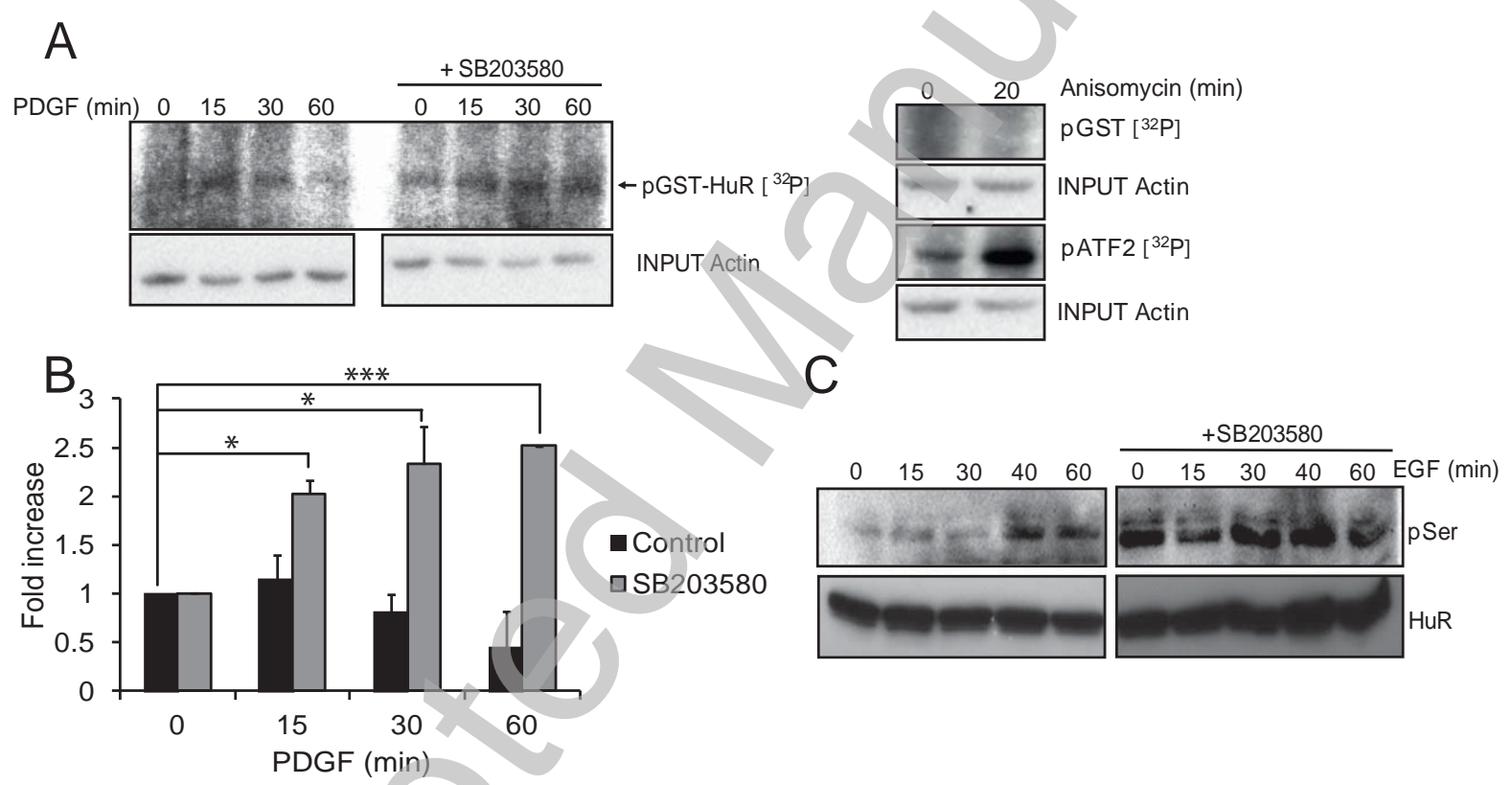


Figure 7

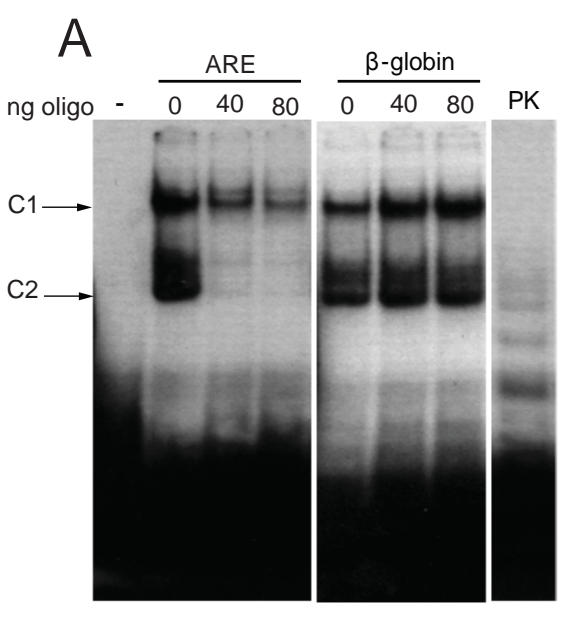

B
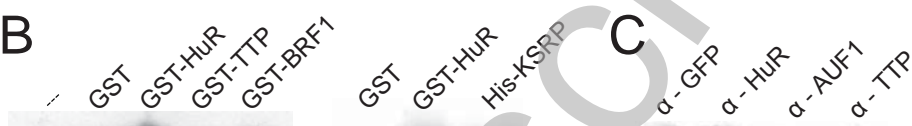

D
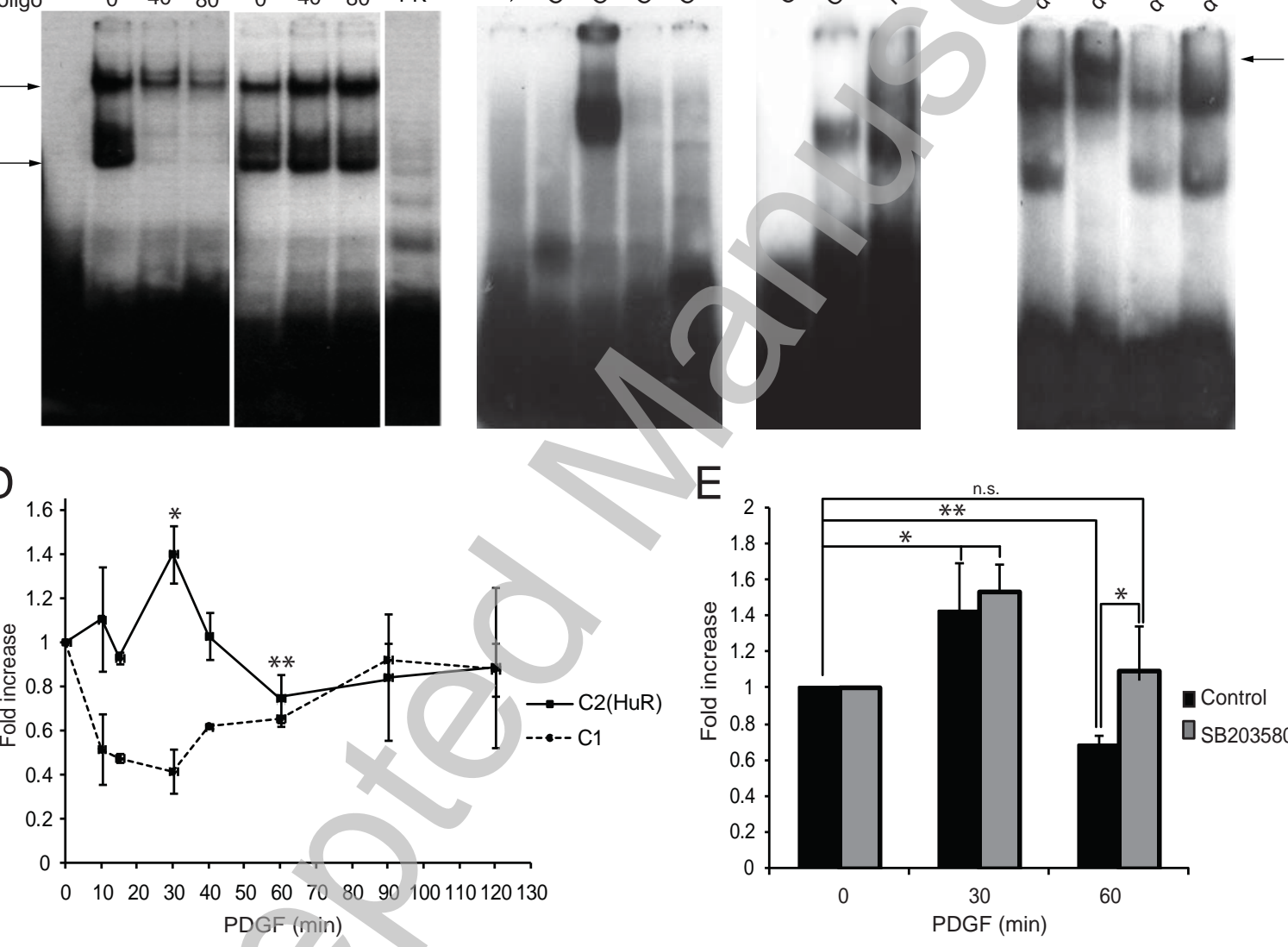

E

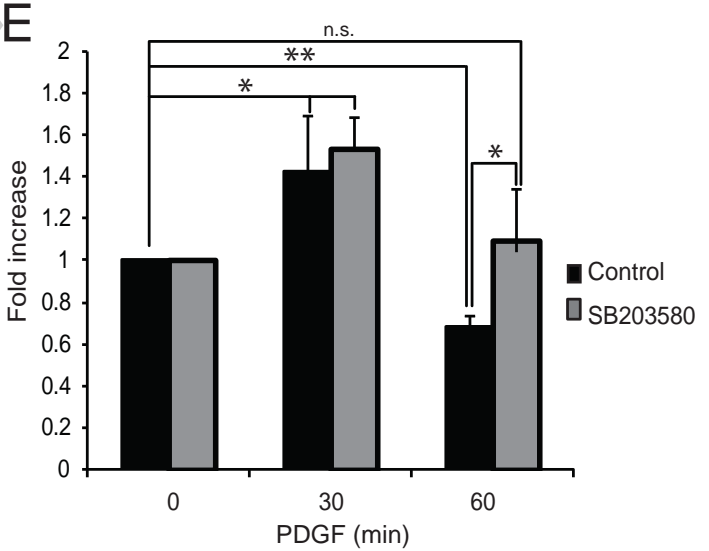

Licenced copy. Copying is not permitted, except with prior permission and as allowed by law. (C) 2015 The Authors Journal compilation (c) 2015 Biochemical Society 


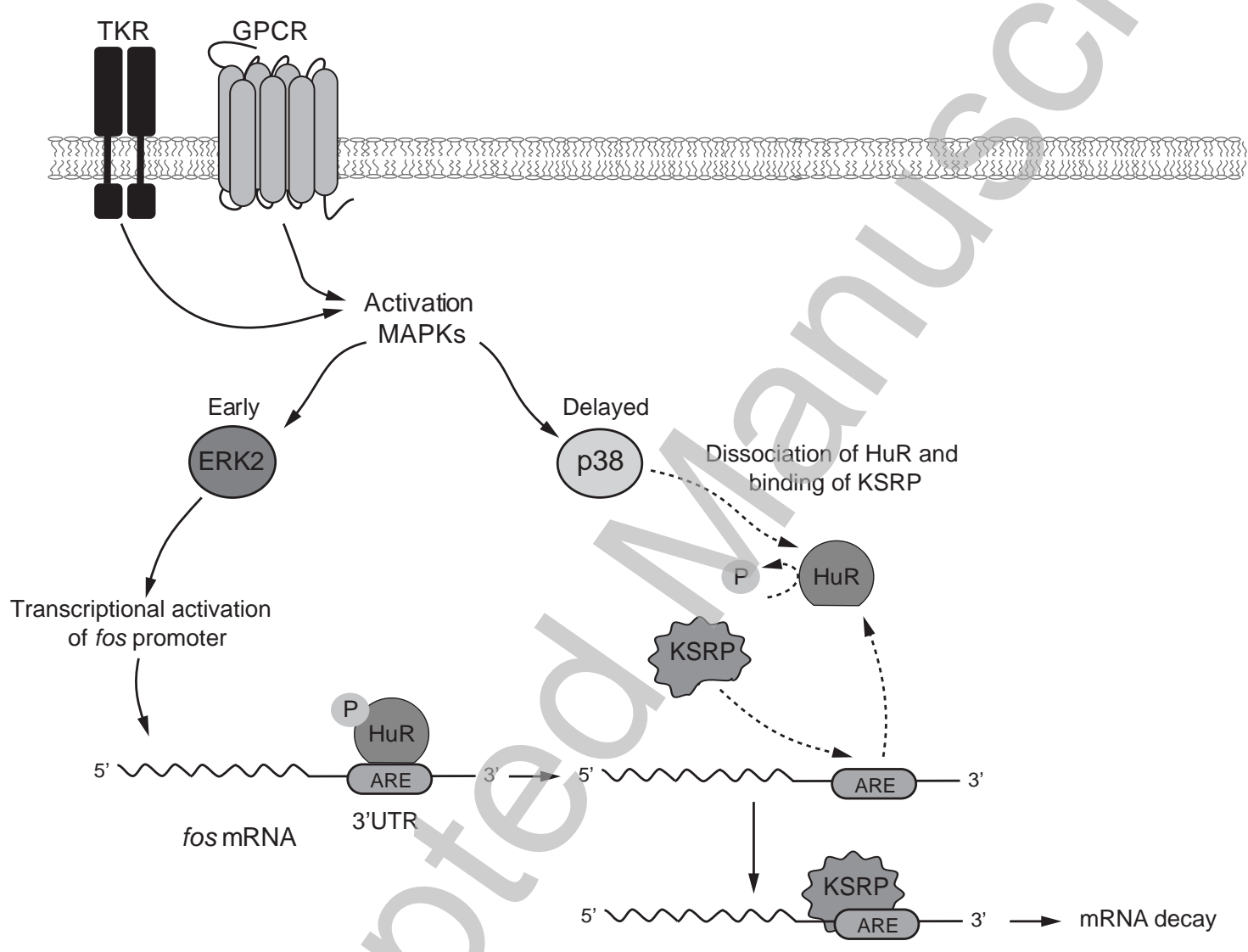

